\title{
Limit theorems for topological invariants of the dynamic multi-parameter simplicial complex
}

\author{
Takashi Owada ${ }^{\mathrm{a}, 1}$, Gennady Samorodnitsky ${ }^{\mathrm{b}, 2}$, Gugan Thoppe ${ }^{\mathrm{c}, *, 1}$ \\ ${ }^{a}$ Department of Statistics, Purdue University, IN, 47907, USA \\ b School of Operations Research and Information Engineering, Cornell University, NY, 14853, USA \\ ${ }^{\mathrm{c}}$ Department of Computer Science and Automation, Indian Institute of Science, Bengaluru, India
}

Received 9 April 2020; received in revised form 3 February 2021; accepted 18 April 2021

Available online 22 April 2021

\begin{abstract}
The topological study of existing random simplicial complexes is non-trivial and has led to several seminal works. However, the applicability of such studies is limited since a single parameter usually governs the randomness in these models. With this in mind, we focus here on the topology of the recently proposed multi-parameter random simplicial complex. In particular, we introduce a dynamic variant of this model and look at how its topology evolves. In this dynamic setup, the temporal evolution of simplices is determined by stationary and possibly non-Markovian processes with a renewal structure. Special cases of this setup include the dynamic versions of the clique complex and the Linial-Meshulam complex. Our key result concerns the regime where the face-count of a particular dimension dominates. We show that the Betti number corresponding to this dimension and the Euler characteristic satisfy a functional strong law of large numbers and a functional central limit theorem. Surprisingly, in the latter result, the limiting process depends only upon the dynamics in the smallest non-trivial dimension.
\end{abstract}

(C) 2021 Elsevier B.V. All rights reserved.

$M S C$ : primary $60 \mathrm{~F} 17$; secondary $55 \mathrm{U} 05 ; 60 \mathrm{C} 05 ; 60 \mathrm{~F} 15$

Keywords: Functional central limit theorem; Functional strong law of large numbers; Betti number; Euler characteristic; Multi-parameter simplicial complex

\footnotetext{
* Corresponding author.

E-mail addresses: owada@purdue.edu (T. Owada), gs18@cornell.edu (G. Samorodnitsky), gthoppe@iisc.ac.in (G. Thoppe).

1 Owada's and Thoppe's research are partially supported by NSF grants DMS-1811428 and DMS-1713012, respectively.

2 Samorodnitsky's research is partially supported by the ARO grant W911NF-18 -10318 at Cornell University.
} 


\section{Introduction}

The classical Erdös-Rényi graph $G(n, p)$ is a random graph on $n$ vertices in which each edge is present with probability $p$ independently. Even in such a simple model, answering topological questions such as the threshold (in terms of the rate of decay of $p=p_{n}$ as $n \rightarrow \infty$ ) for connectivity [6] or for the existence of cycles [17] is completely non-trivial. Not surprisingly then, such a study becomes even more interesting and difficult when posed in the context of random simplicial complexes - the higher dimensional generalizations of random graphs. Our focus in this work is on the general multi-parameter model of combinatorial random simplicial complexes introduced by Costa and Farber [4,5].

A summary of the recent progress made in the study of random complexes generalizing the Erdös-Rényi graph is as follows. The natural complex built over any graph is its clique complex, otherwise known as the flag complex, in which a set of vertices form a face or a simplex if they form a clique in the original graph. The topological properties of the random clique complex built over the Erdös-Rényi graph were studied in [12]. This paper revealed, in particular, the existence of a "dominating dimension", i.e., Betti numbers ${ }^{3}$ of this dimension significantly exceed those of other dimensions, at least on average.

The $k$-dimensional Linial-Meshulam complex is another important extension of the ErdösRényi graph. The $k=2$ case of this model was introduced by Linial and Meshulam [15], which was then extended to general $k$ by Meshulam and Wallach [16]. Here, one starts with a full $(k-1)$-skeleton on $n$ vertices and then adds $k$-simplices with probability $p$ independently. Recently, topological features of the $k$-dimensional Linial-Meshulam complex, with potential $k$-simplices weighted by independent standard uniform random variables, were investigated by Hiraoka and Shirai [10], Hino and Kanazawa [9], Skraba et al. [20], and Fraiman et al. [8].

The multi-parameter model introduced in [4,5] is a generalization of all of these models (see the next section for the formal definition). It was analyzed to some extent in [7], in which it was shown that a dominating dimension exists in this model as well. In this work, we go beyond and examine the topological behavior in this dominating dimension as well as study its deviation from the expected behavior.

Kahle and Meckes [14] did such a study in the context of random clique complexes and proved a central limit theorem for the dominating Betti number. To obtain an even deeper understanding, Thoppe et al. [21] investigated the topological fluctuations in the dynamic variant of this model. Specifically, they considered the setup in which every edge can change its state between being $\mathrm{ON}$ and being OFF, i.e., between being present and being absent, at the transition times of a continuous-time Markov chain. They then derived a functional central limit theorem for the Euler characteristic and the dominating Betti number of the resulting dynamic clique complex.

Within the context of the combinatorial simplicial complexes, few attempts have been made at deriving "process-level" limit theorems for topological invariants (with a few exceptions such as Thoppe et al. [21], Skraba et al. [20], and Fraiman et al. [8]). Our work fills in this gap. We introduce a dynamic variant of the general multi-parameter random simplicial complex and derive a functional strong law of large numbers and a functional central limit theorem for the Euler characteristic and the dominating Betti number. Both of our results are proved in the space $D[0, \infty)$ of right continuous functions with left limits. Additionally, unlike Thoppe et al. [21], we do not assume a Markovian structure for the process according to which the faces of the complex are switched on or off. Instead, the evolution here is determined by a stationary

3 The $k$ th Betti number is a count of "holes" of dimension $k+1$. 
process with a renewal structure. Surprisingly, our key results indicate that the limiting Gaussian process in the central limit theorem depends only upon the dynamics of the faces in the smallest non-trivial dimension, irrespective of the dominating dimension. This happens mainly because the faces in the smallest non-trivial dimension are crucial for the existence of all higher order faces.

The generality of our multi-parameter setup forces us to devise new tools not needed under the random clique complex assumptions of Kahle and Meckes [14] and Thoppe et al. [21]. In the latter case, for example, all Betti numbers of order greater than the dominating dimension vanish with high probability. This is, generally, not the case under our general setup. We solve this difficulty by devising new ways of a much more detailed analysis of these Betti numbers; see Section 7. New coupling arguments play a crucial role as well, especially in the proof of functional strong laws of large numbers. Such coupling arguments enable one to stochastically dominate the face-counts in the dynamic complex by those of a suitably defined static complex, e.g., see (6.5). We believe that such arguments could have applications beyond the present context.

This paper is organized as follows. In Section 2, we construct the dynamic multi-parameter simplicial complex and study some of its elementary properties. A functional central limit theorem for the face counts in this complex is stated in Section 3. Section 4 contains the main theorems for the Euler characteristic and the Betti number in the dominating dimension. The limit theorem for the face counts is proved in Section 5, and the limit theorems for the Euler characteristic are proved in Section 6, while the limit theorems for the Betti numbers in the critical (dominating dimension) are proved in Section 7. Some of the proofs are postponed to the Appendix.

The following notation will be used throughout the paper. The cardinality of a set $A$ will be denoted by $|A|$. The indicator function of an event will be denoted by $\mathbb{1}\{\cdot\}$. For two positive sequences $\left(a_{n}\right)$ and $\left(b_{n}\right)$, the notation $a_{n} \sim b_{n}$ means that $a_{n} / b_{n} \rightarrow 1$ as $n \rightarrow \infty$. The "fat arrow" $\Rightarrow$ is reserved for weak convergence, where the topology is obvious from the context (in this paper it is mostly the Skorohod $J_{1}$-topology on $D[0, \infty)$ ). The stochastic domination of a random variable $X$ by a random variable $Y$ (meaning that $P(X \leq x) \geq P(Y \leq x)$ for all $x$ ) is denoted by $X \stackrel{s t}{\leq} Y$.

\section{The dynamic multi-parameter simplicial complex}

We begin by recalling the original multi-parameter simplicial complex introduced by Costa and Farber [4,5]. Starting with the alphabet $[n]=\{1, \ldots, n\}$ and parameters $\mathbf{p}=\mathbf{p}(n)=$ $\left(p_{1}, \ldots, p_{n-1}\right)$ with $p_{i} \in[0,1], i=1, \ldots, n-1$, one constructs the complex $X([n], \mathbf{p})$ incrementally, one dimension at a time. Specifically, begin with $X([n], \mathbf{p})^{(0)}=[n]$. For $i=1, \ldots, n-1$, once the skeleton ${ }^{4} X([n], \mathbf{p})^{(i-1)}$ has been constructed, add to $X([n], \mathbf{p})$ each $i$-simplex ${ }^{5}$ whose boundary is in $X([n], \mathbf{p})^{(i-1)}$, with probability $p_{i}$ independently of all other potential $i$-simplices. Note that the probabilities in $\mathbf{p}$ may depend on $n$.

Next, we define the "dynamic" version of the multi-parameter simplicial complex with a parameter sequence $\mathbf{p}$. The key ingredient for our construction is a collection of independent stochastic processes

$$
\left(\Delta_{i, A}(t), t \geq 0\right), \quad 1 \leq i \leq n-1, A \in \mathcal{W}_{i},
$$

4 The $i$ th skeleton of a complex consists of all of its faces with dimension less than or equal to $i$.

5 A subset of $[n]$ with cardinality $i+1$. 


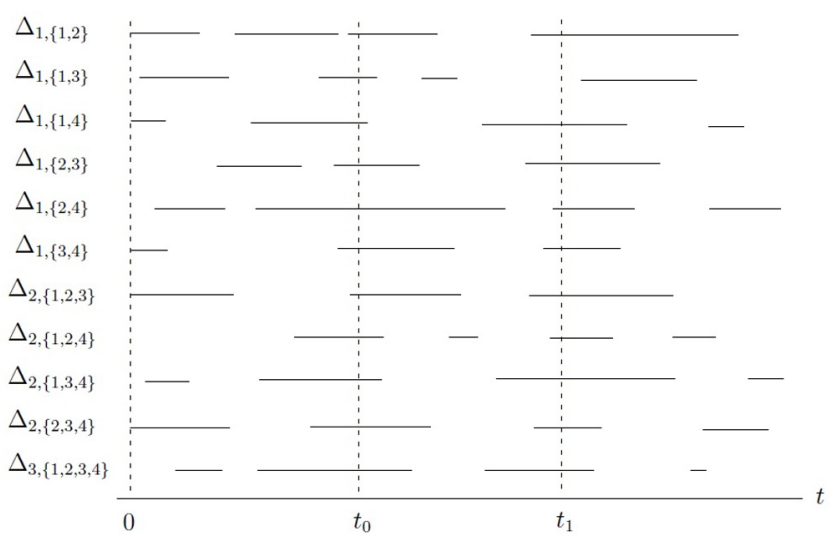

Fig. 1. Eleven independent stochastic processes with $n=4$. Each process stays at an "on" state whenever a line segment appears, and it is at an "off" state if the line segment disappears.

where $\mathcal{W}_{i}:=\{A \subseteq[n]:|A|=i+1\}$. Each of the processes in (2.1) is a $\{0,1\}$-valued stationary process and, for $1 \leq i \leq n-1$ and $A \in \mathcal{W}_{i}$,

$A$ forms an $i$-face at time $t$

$$
\Leftrightarrow \Delta_{\ell, B}(t)=1 \text { for all } \ell \in\{1, \ldots, i\}, B \in \mathcal{W}_{\ell} \text { with } B \subseteq A .
$$

Equivalently, $A$ does not form an $i$-face at time $t$ if and only if $\Delta_{\ell, B}(t)=0$ for some $\ell \in\{1, \ldots, i\}$ and $B \in \mathcal{W}_{\ell}$ with $B \subseteq A$. We say that the process $\Delta_{i, A}$ is "on" at time $t$ if $\Delta_{i, A}(t)=1$, and it is "off" otherwise. We assume that, for each $i \geq 1,\left(\Delta_{i, A}, A \in \mathcal{W}_{i}\right)$ constitutes a family of (independent) processes with a common distribution. We often drop the subscript $A$ when only the dimension $i$ matters.

To give a clear picture of our model, we provide a simple example for $n=4$ in Fig. 1 . In this case, there appears a 3 -face on $[4]=\{1,2,3,4\}$ if and only if the eleven independent processes $\left(\Delta_{i, A}, 1 \leq i \leq 3, A \in \mathcal{W}_{i}\right)$ are all at an "on" state. For example, such a 3-face is present at time $t_{0}$. At time $t_{1}$, the process $\Delta_{1,\{1,3\}}$ is "off", while all the others are "on". Then, the 2 -faces $[1,2,3],[1,3,4]$ and the 3 -face $[1,2,3,4]$ do not appear in the model, whereas all the other 2-faces do exist.

We now model each $\Delta_{i}, i=1, \ldots, n-1$, via a specific $\{0,1\}$-valued stationary renewal process. Let $\left(Z_{j}^{(i)}, j \geq 2\right)$ be a sequence of iid positive random variables with a common distribution function $G_{i}$ and a finite positive mean $\mu_{i}$. The following assumption on the distribution functions $\left(G_{i}\right)$ will be a standing assumption throughout the paper: letting $q:=$ $\min \left\{i \geq 1: p_{i}<1\right\}$, assume that

$$
\text { there is } a>0 \text { such that } G_{i}(a) \leq 1 / 2 \text { for each } i=q, q+1, \ldots \text {. }
$$

Separately, let $\left(I_{j}^{(i)}, j \geq 0\right)$ be a sequence of iid Bernoulli variables with parameter $p_{i}$. Finally, let $D^{(i)}$ be an equilibrium random variable with the distribution

$$
\mathbb{P}\left(D^{(i)} \leq x\right)=\frac{1}{\mu_{i}} \int_{0}^{x}\left(1-G_{i}(y)\right) d y=:\left(G_{i}\right)_{e}(x), \quad x \geq 0 .
$$


All the random objects $\left(Z_{j}^{(i)}\right),\left(I_{j}^{(i)}\right)$, and $D^{(i)}$ are independent. We define a delayed renewal sequence by $S_{0}^{(i)}=0, S_{1}^{(i)}=D^{(i)}$, and

$$
S_{j}^{(i)}=D^{(i)}+\sum_{\ell=2}^{j} Z_{\ell}^{(i)}, \quad j \geq 2,
$$

and the corresponding counting process,

$$
N_{i}(t)=\sum_{j=1}^{\infty} \mathbb{1}\left\{S_{j}^{(i)} \leq t\right\}, \quad t \geq 0 .
$$

Since the first renewal time has the equilibrium distribution given by (2.4), the delayed process $N_{i}$ in (2.6) has stationary increments [19]. In particular, $\mathbb{E}\left(N_{i}(t)\right)=t / \mu_{i}$. We finally define

$$
\Delta_{i}(t):=\sum_{j=0}^{\infty} \mathbb{1}\left\{S_{j}^{(i)} \leq t<S_{j+1}^{(i)}\right\} I_{j}^{(i)}, \quad t \geq 0 .
$$

Definition 2.1. The dynamic multi-parameter simplicial complex $(X([n], \mathbf{p} ; t), t \geq 0)$ on $n$ vertices is defined by (2.2). For each dimension $i$, the temporal evolution of the $i$-dimensional faces is determined by the independent processes $\left(\Delta_{i, A}, 1 \leq i \leq n-1, A \in \mathcal{W}_{i}\right)$ described in (2.7).

Remark 2.2. As stated below in Lemma 2.4, $\Delta_{i}$ is a stationary process for every $i$ which implies that $(X([n], \mathbf{p} ; t), t \geq 0)$ itself is stationary. In fact, for each $t \geq 0, X([n], \mathbf{p} ; t)$ has the same distribution as that of the static multi-parameter simplicial complex in [4,5].

Remark 2.3. If $\mathbf{p}=(p, 1,1, \ldots)$ and $G_{1}(x)=1-e^{-\lambda x}, x \geq 0$ for some $\lambda>0$, then $X([n], \mathbf{p} ; t)$ is a reparametrization of the dynamic clique complex, for which the evolution of the edges is determined by the $\{0,1\}$-valued stationary continuous-time Markov chain [21].

The next result formally records the fact that, for each $i, \Delta_{i}$ is a stationary process. It also states and proves a couple of useful properties concerning it. In particular, it shows that if $p_{i}$ is small, then $\Delta_{i}$ is off most of the times.

Lemma 2.4. (i) For every $i \in\{1, \ldots, n-1\},\left(\Delta_{i}(t), t \geq 0\right)$ is a stationary process with $\mathbb{P}\left(\Delta_{i}(t)=1\right)=p_{i}$. In addition,

$$
\mathbb{P}\left(\Delta_{i}(t)=1 \mid \Delta_{i}(0)=1\right)=1-\left(1-p_{i}\right)\left(G_{i}\right)_{e}(t), \quad t \geq 0 .
$$

(ii) For every $i \geq q$ and $T>0$,

$$
\mathbb{P}\left(\sup _{0 \leq t \leq T} \Delta_{i}(t)=1\right) \leq p_{i}\left(1+\left(1-p_{i}\right) \frac{\left(G_{i}\right)_{e}(T)}{1-G_{i}(T)}\right) .
$$

Proof. The first statement in part (i) is obvious, because the process $N_{i}(t)$ has stationary increments. For the second one,

$$
\begin{aligned}
\mathbb{P}\left(\Delta_{i}(t)=1 \mid \Delta_{i}(0)=1\right) & =\mathbb{P}\left(0 \leq t<D^{(i)}\right)+p_{i} P\left(t \geq D^{(i)}\right) \\
& =1-\left(1-p_{i}\right)\left(G_{i}\right)_{e}(t) .
\end{aligned}
$$


For Part (ii), denote

$$
K=N_{i}(T)=\max \left\{j \geq 1: S_{j}^{(i)} \leq T\right\} \quad\left(K=0 \text { if } S_{1}^{(i)}>T\right) .
$$

Then,

$$
\begin{aligned}
\mathbb{P}\left(\sup _{0 \leq t \leq T} \Delta_{i}(t)=1\right) & =p_{i}+\mathbb{P}\left(\Delta_{i}(0)=0, \sup _{0<t \leq T} \Delta_{i}(t)=1\right) \\
& =p_{i}+\left(1-p_{i}\right) \mathbb{E}\left[1-\left(1-p_{i}\right)^{K}\right] .
\end{aligned}
$$

It is clear that $K$ is dominated by

$$
K^{\prime}:= \begin{cases}\min \left\{j \geq 2: Z_{j}^{(i)}>T\right\}-1 & \text { if } D^{(i)} \leq T \\ 0 & \text { if } D^{(i)}>T .\end{cases}
$$

Evaluating the above expression with $K$ replaced by $K^{\prime}$ gives us (2.8).

Sometimes we will also impose the following additional assumption on the distributions $\left(G_{i}\right)$.

$$
c:=\sup _{i \geq q} \sup _{h>0,0 \leq y \leq 1} \frac{G_{i}(y+h)-G_{i}(y)}{h^{\gamma}}<\infty \text { for some } 0<\gamma \leq 1,
$$

Note that (2.9) holds if $G_{i}$ 's have a common bounded density function (such as an exponential density).

Under this additional assumption, we have the following estimates.

Lemma 2.5. Assume (2.9). Then for all $0 \leq r<s<t \leq 1$,

$$
\mathbb{P}\left(\Delta_{i}(r)=0, \Delta_{i}(s)=1, \Delta_{i}(t)=0\right) \leq \frac{2 c}{a} p_{i}(t-r)^{1+\gamma}
$$

and

$$
\mathbb{P}\left(\Delta_{i}(r)=1, \Delta_{i}(s)=0, \Delta_{i}(t)=1\right) \leq \frac{2 c}{a} p_{i}^{2}(t-r)^{1+\gamma} .
$$

Proof. Rewrite (2.10) as

$$
p_{i} \mathbb{P}\left(\Delta_{i}(r)=0, \Delta_{i}(t)=0 \mid \Delta_{i}(s)=1\right) \leq p_{i} \mathbb{P}\left(A_{i}(s) \leq s-r, R_{i}(s) \leq t-s\right),
$$

where $A_{i}$ and $R_{i}$ are respectively, the age and the residual lifetime of a renewal process (2.6) with the interarrival distribution $G_{i}$. It then follows from standard calculation in renewal theory (see e.g., [18]) that

$$
\begin{aligned}
\mathbb{P}\left(A_{i}(s) \leq s-r, R_{i}(s) \leq t-s\right) & =\mathbb{P}\left(r \leq S_{N_{i}(s)}^{(i)}, S_{N_{i}(s)+1}^{(i)} \leq t\right) \\
& =\frac{1}{\mu_{i}} \int_{0}^{s-r}\left(G_{i}(y+t-s)-G_{i}(y)\right) d y \\
& \leq \frac{2 c}{a}(s-r)(t-s)^{\gamma} .
\end{aligned}
$$

The last inequality comes from (2.3) and (2.9). The argument for (2.11) is similar; since the process $\Delta_{i}$ is now required to be "on" in two distinct time intervals, $p_{i}$ in (2.10) is replaced by $p_{i}^{2}$. 
Recall that the probabilities in $\mathbf{p}$ for the dynamic multi-parameter simplicial complex $X([n], \mathbf{p} ; t)$ may depend on $n$. In the sequel, following Costa and Farber [5], we "couple" p with $n$ in a particular way: we set $p_{i}=n^{-\alpha_{i}}, \alpha_{i} \in[0, \infty]$ for $i=1,2, \ldots$. Accordingly, we can work with an infinite sequence $\boldsymbol{\alpha}=\left(\alpha_{1}, \alpha_{2}, \ldots\right)$, independent of $n$, to control the rates at which the entries in $\mathbf{p}$ decay. Below, we introduce some additional terms and notation, which we try to keep as consistent as possible with those in [5].

Let

$$
\psi_{j}(\boldsymbol{\alpha})=\sum_{i=1}^{j}\left(\begin{array}{l}
j \\
i
\end{array}\right) \alpha_{i}, \quad j \geq 1
$$

By convention, we set $\left(\begin{array}{l}j \\ i\end{array}\right)=0$ whenever $j<i$. Note that $\psi_{j}(\boldsymbol{\alpha})$ is non-decreasing in $j$, i.e., $\psi_{i}(\boldsymbol{\alpha}) \leq \psi_{j}(\boldsymbol{\alpha})$ for each $\boldsymbol{\alpha}$ and $i \leq j$. We also let

$$
\tau_{j}(\boldsymbol{\alpha}):=j+1-\sum_{i=1}^{j} \psi_{i}(\boldsymbol{\alpha})=j+1-\sum_{i=1}^{j}\left(\begin{array}{c}
j+1 \\
i+1
\end{array}\right) \alpha_{i}, \quad 1 \leq j \leq n-1 .
$$

Additionally, we consider the following sets of parameters:

$$
\mathcal{D}_{j}:=\left\{\boldsymbol{\alpha}: \psi_{j}(\boldsymbol{\alpha})<1<\psi_{j+1}(\boldsymbol{\alpha})\right\}
$$

for $j \geq 1$ and $\mathcal{D}_{0}:=\left\{\boldsymbol{\alpha}: \psi_{1}(\boldsymbol{\alpha})>1\right\}$.

Recalling the notation $q=q(\boldsymbol{\alpha})=\min \left\{i \geq 1: \alpha_{i}>0\right\}$ in (2.3), note that

$$
\psi_{j}(\boldsymbol{\alpha})=0, \quad \tau_{j}(\boldsymbol{\alpha})=j+1, \quad j=1, \ldots, q-1 .
$$

Importantly, if $\boldsymbol{\alpha} \in \mathcal{D}_{k}$ for some $k \geq q$, then

$$
0<\psi_{q}(\boldsymbol{\alpha})<\cdots<\psi_{k}(\boldsymbol{\alpha})<1<\psi_{k+1}(\boldsymbol{\alpha})<\ldots,
$$

so that,

$$
q=\tau_{q-1}(\boldsymbol{\alpha})<\tau_{q}(\boldsymbol{\alpha})<\cdots<\tau_{k}(\boldsymbol{\alpha})>\tau_{k+1}(\boldsymbol{\alpha})>\ldots
$$

In this case, the index $k$ is referred to as the critical dimension. Note that $\tau_{j}(\boldsymbol{\alpha}), j \geq k+1$, can be negative. Observe also that, for $j>k$,

$$
\begin{aligned}
\tau_{j}(\boldsymbol{\alpha})-\left(\tau_{j+1}(\boldsymbol{\alpha})+\alpha_{j+1}\right) & =-1+\sum_{i=1}^{j}\left(\left(\begin{array}{l}
j+2 \\
i+1
\end{array}\right)-\left(\begin{array}{l}
j+1 \\
i+1
\end{array}\right)\right) \alpha_{i} \\
& =-1+\sum_{i=1}^{j}\left(\begin{array}{c}
j+1 \\
i
\end{array}\right) \alpha_{i}>-1+\psi_{j}(\boldsymbol{\alpha})>0 .
\end{aligned}
$$

\section{Limit theorems for the face counts}

We consider the dynamic multi-parameter simplicial complex $(X([n], \mathbf{p} ; t), t \geq 0)$ constructed in the previous section. Our basic assumption from now on will be that

$$
\alpha \in \mathcal{D}_{k} \text { for some } k \geq q .
$$

Let $\beta_{j, n}(t):=\beta_{j, n}(X([n], \mathbf{p} ; t))$ be the $j$ th (reduced) Betti number of the complex at time $t$. Note that $\left(\beta_{j, n}(t), t \geq 0\right)$ is a stationary process. We will often use $\beta_{j, n}$ to mean $\beta_{j, n}(0)$. Similarly, we let $\chi_{n}(t)$ denote the Euler characteristic of the complex at time $t$. Then, $\left(\chi_{n}(t), t \geq 0\right)$ also is a stationary process, and $\chi_{n}$ will be used to denote $\chi_{n}:=\chi_{n}(0)$. Recall 
that our goal is to establish functional strong laws of large numbers (SLLN) and functional central limit theorems (FCLT) for the Euler characteristic and the Betti number in the critical dimension $k$ of the dynamic multi-parameter simplicial complex. This section is of preparatory nature and deals with the face counts of the complex.

We write the face counts in dimension $j$ as

$$
f_{j, n}(t)=\sum_{\sigma \subset[n],|\sigma|=j+1} \mathbb{1}\{\sigma \text { forms a } j \text {-face in } X([n], \mathbf{p} ; t)\}=: \sum_{\sigma \subset[n],|\sigma|=j+1} \xi_{\sigma}(t), \quad t \geq 0 .
$$

Once again, let $\xi_{\sigma}:=\xi_{\sigma}(0)$. As in [14] and [21], we analyze the face counts first, and then relate them to the Euler characteristic and the Betti numbers through the relations

$$
\chi_{n}(t):=\sum_{j=0}^{n-1}(-1)^{j} f_{j, n}(t), \quad t \geq 0,
$$

and

$$
\chi_{n}(t):=1+\sum_{j=0}^{n-1}(-1)^{j} \beta_{j, n}(t), \quad t \geq 0 .
$$

We start with the asymptotic behavior of the expected value and the covariances of the face counts. Note that not all results below require the assumption (3.1).

Proposition 3.1. For any $j \geq 1$, we have

$$
\mathbb{E}\left(f_{j, n}\right) \sim \frac{n^{\tau_{j}(\alpha)}}{(j+1) !}, \quad n \rightarrow \infty .
$$

Furthermore, for $j \geq q$ and $0 \leq s \leq t<\infty$, we have

$$
\begin{aligned}
\operatorname{Cov}\left(f_{j, n}(t), f_{j, n}(s)\right) \sim & \frac{n^{2 \tau_{j}(\boldsymbol{\alpha})-\tau_{q}(\boldsymbol{\alpha})}}{(q+1) !((j-q) !)^{2}}\left(1-\left(G_{q}\right)_{e}(t-s)\right) \\
& \vee \frac{n^{\tau_{j}(\boldsymbol{\alpha})}}{(j+1) !} \prod_{i=q}^{j}\left(1-\left(1-p_{i}\right)\left(G_{i}\right)_{e}(t-s)\right)^{(j+1)}
\end{aligned}
$$

as $n \rightarrow \infty$, where $a \vee b=\max \{a, b\}$ for $a, b \in \mathbb{R}$. In particular, if (3.1) holds, then

$$
\operatorname{Cov}\left(f_{k, n}(t), f_{k, n}(s)\right) \sim \frac{n^{2 \tau_{k}(\boldsymbol{\alpha})-\tau_{q}(\boldsymbol{\alpha})}}{(q+1) !((k-q) !)^{2}}\left(1-\left(G_{q}\right)_{e}(t-s)\right), \quad n \rightarrow \infty .
$$

Remark 3.2. For $j<q, f_{j, n}(t)$ is, of course, nonrandom, so in this case, $\operatorname{Cov}\left(f_{j, n}(t), f_{j, n}(s)\right)=0$.

Proof. The asymptotics of the mean face count is easy to obtain. In fact,

$$
\mathbb{E}\left(f_{j, n}\right)=\left(\begin{array}{c}
n \\
j+1
\end{array}\right) \prod_{i=q}^{j} p_{i}^{\left(\begin{array}{c}
j+1 \\
i+1
\end{array}\right)}=\left(\begin{array}{c}
n \\
j+1
\end{array}\right) n^{\tau_{j}(\boldsymbol{\alpha})-(j+1)} \sim \frac{n^{\tau_{j}(\boldsymbol{\alpha})}}{(j+1) !} \quad \text { as } n \rightarrow \infty .
$$

For the covariances, we write

$$
\mathbb{E}\left(f_{j, n}(t) f_{j, n}(s)\right)=\sum_{\ell=0}^{j+1} \mathbb{E}\left(\sum_{\substack{\sigma \subset[n] \\|\sigma|=j+1}} \sum_{\substack{\tau \subset[n] \\|\tau|=j+1,|\sigma \cap \tau|=\ell}} \xi_{\sigma}(t) \xi_{\tau}(s)\right)
$$




$$
=\sum_{\ell=0}^{j+1}\left(\begin{array}{c}
n \\
j+1
\end{array}\right)\left(\begin{array}{c}
j+1 \\
\ell
\end{array}\right)\left(\begin{array}{c}
n-j-1 \\
j+1-\ell
\end{array}\right) \mathbb{E}\left(\xi_{\sigma}(t) \xi_{\tau}(s)\right) \mathbb{1}\{|\sigma \cap \tau|=\ell\} .
$$

If $\ell \in\{0,1, \ldots, q\}$, all faces of $\sigma \cap \tau$ exist with probability one; thus,

$$
\mathbb{E}\left(\xi_{\sigma}(t) \xi_{\tau}(s)\right) \mathbb{1}\{|\sigma \cap \tau|=\ell\}=\left(\prod_{i=q}^{j} p_{i}^{\left(\begin{array}{l}
j+1 \\
i+1
\end{array}\right)}\right)^{2}=n^{2 \tau_{j}(\boldsymbol{\alpha})-2(j+1)} .
$$

On the other hand, if $\ell \in\{q+1, \ldots, j+1\}$, we have

$$
\begin{aligned}
\mathbb{E}\left(\xi_{\sigma}(t) \xi_{\tau}(s)\right) \mathbb{1}\{|\sigma \cap \tau|=\ell\} & =\prod_{i=q}^{j} p_{i}^{\left(\begin{array}{c}
j+1 \\
i+1
\end{array}\right)} \times \prod_{i=q}^{j} \mathbb{P}\left(\Delta_{i}(t)=1 \mid \Delta_{i}(s)=1\right)^{\left(\begin{array}{c}
\ell \\
i+1
\end{array}\right)} \\
& \times \prod_{i=q}^{j} p_{i}^{\left(\begin{array}{c}
j+1 \\
i+1
\end{array}\right)-\left(\begin{array}{c}
\ell \\
i+1
\end{array}\right)} \\
& =: A_{n} \times B_{n} \times C_{n} .
\end{aligned}
$$

Here, $A_{n}$ is the probability of $\tau$ spanning a $j$-face at time $s$, while $B_{n}$ is the conditional probability that all faces of $\sigma \cap \tau$ are present at time $t$, given that $\tau$ spans a $j$-face at time $s$. Finally, $C_{n}$ is the conditional probability of $\sigma$ forming a $j$-face at time $t$, given that all faces of $\sigma \cap \tau$ are present at time $t$. Calculating the product of three terms via Lemma 2.4,

$$
A_{n} \times B_{n} \times C_{n}=n^{2 \tau_{j}(\boldsymbol{\alpha})-\tau_{\ell-1}(\boldsymbol{\alpha})-2(j+1)+\ell} \prod_{i=q}^{j}\left(1-\left(1-p_{i}\right)\left(G_{i}\right)_{e}(t-s)\right)^{\left(\begin{array}{l}
\ell \\
i+1
\end{array}\right)} .
$$

By the stationarity of face counts, together with (3.5), we have that

$$
\begin{aligned}
\mathbb{E}\left(f_{j, n}(t)\right) \mathbb{E}\left(f_{j, n}(s)\right) & =\left(\mathbb{E}\left(f_{j, n}\right)\right)^{2}=\left(\begin{array}{c}
n \\
j+1
\end{array}\right)^{2} n^{2 \tau_{j}(\boldsymbol{\alpha})-2(j+1)} \\
& =\sum_{\ell=0}^{j+1}\left(\begin{array}{c}
n \\
j+1
\end{array}\right)\left(\begin{array}{c}
j+1 \\
\ell
\end{array}\right)\left(\begin{array}{c}
n-j-1 \\
j+1-\ell
\end{array}\right) n^{2 \tau_{j}(\boldsymbol{\alpha})-2(j+1)} .
\end{aligned}
$$

Combining all these results yields that, as $n \rightarrow \infty$,

$$
\begin{aligned}
& \operatorname{Cov}\left(f_{j, n}(t), f_{j, n}(s)\right)= \sum_{\ell=q+1}^{j+1}\left(\begin{array}{c}
n \\
j+1
\end{array}\right)\left(\begin{array}{c}
j+1 \\
\ell
\end{array}\right)\left(\begin{array}{c}
n-j-1 \\
j+1-\ell
\end{array}\right) n^{2 \tau_{j}(\boldsymbol{\alpha})-\tau_{\ell-1}(\boldsymbol{\alpha})-2(j+1)+\ell} \\
& \times\left\{\prod_{i=q}^{j}\left(1-\left(1-p_{i}\right)\left(G_{i}\right)_{e}(t-s)\right)^{\left(\begin{array}{c}
\ell \\
i+1
\end{array}\right)}-n^{\tau_{\ell-1}(\boldsymbol{\alpha})-\ell}\right\} \\
& \sim \sum_{\ell=q+1}^{j+1} \frac{n^{2 \tau_{j}(\boldsymbol{\alpha})-\tau_{\ell-1}(\boldsymbol{\alpha})}}{\ell !((j+1-\ell) !)^{2}} \prod_{i=q}^{\ell-1}\left(1-\left(1-p_{i}\right)\left(G_{i}\right)_{e}(t-s)\right)^{\left(\begin{array}{c}
\ell \\
i+1
\end{array}\right)} \\
& \sim \frac{n^{2 \tau_{j}(\boldsymbol{\alpha})-\tau_{q}(\boldsymbol{\alpha})}}{(q+1) !((j-q) !)^{2}}\left(1-\left(G_{q}\right)_{e}(t-s)\right) \\
&\left.\vee \frac{n^{\tau_{j}(\boldsymbol{\alpha})}}{(j+1) !} \prod_{i=q}^{j}\left(1-\left(1-p_{i}\right)\left(G_{i}\right)_{e}(t-s)\right)^{(j+1} i+1\right)
\end{aligned}
$$


where the last equivalence comes from the fact that $\left(\tau_{\ell}(\boldsymbol{\alpha}), \ell \geq q\right)$ is a sequence that increases for $\ell \leq k$ and then decreases. For the derivation of (3.4), use the fact that $2 \tau_{k}(\boldsymbol{\alpha})-\tau_{q}(\boldsymbol{\alpha}) \geq$ $\tau_{k}(\boldsymbol{\alpha})$.

Remark 3.3. It follows immediately from the proposition that, under the assumption (3.1), for every $j \neq k$,

$$
\lim _{n \rightarrow \infty} \frac{\mathbb{E}\left(f_{j, n}\right)}{\mathbb{E}\left(f_{k, n}\right)}=\lim _{n \rightarrow \infty} \frac{\operatorname{Var}\left(f_{j, n}\right)}{\operatorname{Var}\left(f_{k, n}\right)}=0 .
$$

That is, the face counts in the critical dimension dominate those in the other dimensions both in their means and their variances.

The following corollary will be useful in the sequel. Since time parameter plays no role due to stationarity, we remove it to simplify the notation. Denote

$$
M(\boldsymbol{\alpha})=\min \left\{i: \tau_{i}(\boldsymbol{\alpha})<0\right\}
$$

this is a finite number since $\tau_{i}(\boldsymbol{\alpha}) \rightarrow-\infty$ as $i \rightarrow \infty$.

Corollary 3.4. As $n \rightarrow \infty$,

$$
\sum_{j=M(\boldsymbol{\alpha})}^{\infty} \mathbb{E}\left(f_{j, n}\right) \rightarrow 0
$$

Proof. It follows from (3.5) that

$$
\mathbb{E}\left(f_{j, n}\right) \leq n^{\tau_{j}(\boldsymbol{\alpha})} \leq\left(\frac{1}{n^{\beta}}\right)^{j+1},
$$

where

$$
\beta=\inf _{j \geq M(\boldsymbol{\alpha})}\left[-\tau_{j}(\boldsymbol{\alpha}) /(j+1)\right] .
$$

Note that $\beta>0$, since $\tau_{j}(\boldsymbol{\alpha})<0$ for all $j \geq M(\boldsymbol{\alpha})$, and

$$
\lim _{j \rightarrow \infty} \frac{-\tau_{j}(\boldsymbol{\alpha})}{j+1}=\lim _{j \rightarrow \infty}\left\{\sum_{i=1}^{j}\left(\begin{array}{l}
j \\
i
\end{array}\right) \frac{\alpha_{i}}{i+1}-1\right\} \geq \lim _{j \rightarrow \infty}\left\{\left(\begin{array}{l}
j \\
q
\end{array}\right) \frac{\alpha_{q}}{q+1}-1\right\}=\infty .
$$

Hence,

$$
\sum_{j=M(\boldsymbol{\alpha})}^{\infty} \mathbb{E}\left(f_{j, n}\right) \leq \sum_{j=M(\boldsymbol{\alpha})}^{\infty}\left(\frac{1}{n^{\beta}}\right)^{j+1} \rightarrow 0, \quad n \rightarrow \infty,
$$

as desired.

As stated below, the face counts in the critical dimension $k$ turn out to satisfy a functional central limit theorem. The limit turns out to be a stationary Gaussian process whose covariance function is given by the limit in (3.4). Specifically, let $\left(Z_{k}(t), t \geq 0\right)$ be a zero-mean stationary Gaussian process with covariance function

$$
R_{k}(t)=\mathbb{E}\left(Z_{k}(t) Z_{k}(0)\right)=1-\left(G_{q}\right)_{e}(t), \quad t \geq 0 .
$$

The basic sample path properties of this process are described in the next proposition. 
Proposition 3.5. The process $Z_{k}$ admits a continuous version, whose sample paths are $\delta$-Hölder continuous for any $\delta \in(0,1 / 2)$.

Proof. Since $Z_{k}$ is a stationary Gaussian process and

$$
\mathbb{E}\left[\left(Z_{k}(t)-Z_{k}(s)\right)^{2}\right]=2\left(G_{q}\right)_{e}(|t-s|) \leq \frac{2}{\mu_{q}}|t-s|,
$$

the claim follows from the Kolmogorov continuity criterion.

The statement below is a FCLT for the face counts in the critical dimension $k$. We view $f_{k, n}(\cdot)$ as a (piecewise constant) random element of $D[0, \infty)$, the space of right continuous functions with left limits, which is equipped with the Skorohod $J_{1}$-topology.

Proposition 3.6. Assume (3.1). Then, as $n \rightarrow \infty$,

$$
\left(\frac{f_{k, n}(t)-\mathbb{E}\left(f_{k, n}\right)}{\sqrt{\operatorname{Var}\left(f_{k, n}\right)}}, t \geq 0\right) \Rightarrow\left(Z_{k}(t), t \geq 0\right)
$$

in the sense of convergence of the finite-dimensional distributions. If the assumption (2.9) is satisfied then (3.9) also holds in the sense of weak convergence in the $J_{1}$-topology on $D[0, \infty)$.

The proof is deferred to Section 5.

Remark 3.7. It is interesting and, initially, unexpected that only the state change distribution $G_{q}$ in the lowest nontrivial dimension $q$ contributes to the asymptotics of the face counts in the critical dimension. This is due to the fact that the "flipping" of a $q$-simplex from "on" to "off" or vice versa affects the distribution of $k$-simplices more than does any flipping in a different dimension. Note that if $G_{q}$ is exponential with mean $1 / \lambda$, then $R_{k}(t)=e^{-\lambda t}$ and $Z_{k}$ is the Ornstein-Uhlenbeck Gaussian process, as in [21].

\section{FCLT for topological invariants}

In this section, we present the main results of this paper: the functional SLLN and the FCLT for the Euler characteristic and the Betti numbers in the critical dimension. We defer the proofs to Sections 6 and 7.

We start with the strong laws of large numbers.

Theorem 4.1. Assume (3.1). Then, as $n \rightarrow \infty$,

$$
\left(\frac{\chi_{n}(t)}{n^{\tau_{k}(\alpha)}}, t \geq 0\right) \rightarrow \frac{(-1)^{k}}{(k+1) !} \text { a.s. }
$$

and

$$
\left(\frac{\beta_{k, n}(t)}{n^{\tau_{k}(\alpha)}}, t \geq 0\right) \rightarrow \frac{1}{(k+1) !} \text { a.s. }
$$

in the $J_{1}$-topology on $D[0, \infty)$, where the right hand sides of (4.1) and (4.2) are viewed as constant elements of $D[0, \infty)$.

After stating the functional strong law of large numbers, we proceed, as it is frequently done, with the functional central limit theorem. Note the similarity with the corresponding limit theorem for the face counts in Proposition 3.6. 
Theorem 4.2. Assume (3.1). Then, as $n \rightarrow \infty$,

$$
\left(\frac{\chi_{n}(t)-\mathbb{E}\left(\chi_{n}\right)}{\sqrt{\operatorname{Var}\left(f_{k, n}\right)}}, t \geq 0\right) \Rightarrow\left(Z_{k}(t), t \geq 0\right)
$$

and

$$
\left(\frac{\beta_{k, n}(t)-\mathbb{E}\left(\beta_{k, n}\right)}{\sqrt{\operatorname{Var}\left(f_{k, n}\right)}}, t \geq 0\right) \Rightarrow\left(Z_{k}(t), t \geq 0\right)
$$

in the sense of convergence of the finite-dimensional distributions.

In addition, assume (2.9) and

$$
\tau_{k}(\boldsymbol{\alpha})-\frac{\tau_{q}(\boldsymbol{\alpha})}{2}>\tau_{k+1}(\boldsymbol{\alpha})
$$

Then, (4.3) and (4.4) also hold in the sense of weak convergence in the $J_{1}$-topology on $D[0, \infty)$.

Remark 4.3. By Proposition 3.1, (4.3) can be restated as

$$
\left(\frac{\chi_{n}(t)-\mathbb{E}\left(\chi_{n}\right)}{n^{\tau_{k}(\boldsymbol{\alpha})-\tau_{q}(\boldsymbol{\alpha}) / 2}}, t \geq 0\right) \Rightarrow\left(\{(q+1) !\}^{1 / 2}(k-q) ! Z_{k}(t), t \geq 0\right)
$$

A similar reformulation is possible for (4.4).

Remark 4.4. We think that (2.9) alone is sufficient for weak convergence in the $J_{1}$-topology on $D[0, \infty)$ in (4.3) and (4.4). We have chosen to assume (4.5) in order to simplify an already long and technical argument.

Example 4.5. The dynamic variants of the Linial-Meshulam complex and the clique complex are special cases of our model. An explicit form of Theorem 4.2 is stated here for these two setups.

The Linial-Meshulam simplicial complex (see $[15,16]$ ) corresponds, in our description, to $\boldsymbol{\alpha}=\left(0, \ldots, 0, \alpha_{k}, \infty, \infty, \ldots\right)$, with $0<\alpha_{k}<1$ in some position $k \geq 2$. This $k$ is then the critical dimension with $q=k$, and $\tau_{k}(\boldsymbol{\alpha})=k+1-\alpha_{k}$. Furthermore, (3.1) holds. If $X([n], \mathbf{p} ; t)$ is the dynamic Linial-Meshulam complex, then Theorem 4.2 says that

$$
\left(\frac{\chi_{n}(t)-\mathbb{E}\left(\chi_{n}\right)}{\sqrt{n^{k+1-\alpha_{k}}}}, t \geq 0\right) \Rightarrow\left(\{(k+1) !\}^{1 / 2} Z_{k}(t), t \geq 0\right),
$$

at least in the sense of finite-dimensional distributions. 
Consider now the dynamic clique complex, for which $\boldsymbol{\alpha}=\left(\alpha_{1}, 0,0, \ldots\right)$ with $0<\alpha_{1}<1$ and $\alpha_{1} \neq 1 / m$ for any $m \in \mathbb{N}$. Then, $q=1$ and the critical dimension is $k=\left\lfloor 1 / \alpha_{1}\right\rfloor \geq q$. Once again, (3.1) holds. Here, $\tau_{k}(\boldsymbol{\alpha})=k+1-\left(\begin{array}{c}k+1 \\ 2\end{array}\right) \alpha_{1}$ and $\tau_{q}(\boldsymbol{\alpha})=2-\alpha_{1}$. Now, Theorem 4.2 says that

$$
\left(\frac{\chi_{n}(t)-\mathbb{E}\left(\chi_{n}\right)}{n^{k-\left(\begin{array}{c}
k+1 \\
2
\end{array}\right) \alpha_{1}+\alpha_{1} / 2}}, t \geq 0\right) \Rightarrow\left(\sqrt{2}(k-1) ! Z_{k}(t), t \geq 0\right),
$$

once again, at least in the finite-dimensional distributions.

For both models, we also obtain corresponding results for the Betti numbers in the critical dimension. In the dynamic clique complex, if $G_{1}$ is an exponential distribution, then, as mentioned above, $Z_{k}$ is a zero-mean stationary Ornstein-Uhlenbeck Gaussian process, as in [21].

As for the technical conditions for tightness, in the dynamic Linial-Meshulam complex, we only need to check (2.9) just for $i=k$, while (4.5) always holds as $\tau_{k+1}(\boldsymbol{\alpha})=-\infty$. In the case of a dynamic clique complex, one needs to check (2.9) just for $i=1$. On the other hand, (4.5) reduces to $\alpha_{1}>4 /(2 k+3)$, implying that the corresponding functional convergence follows only when $4 / 5<\alpha_{1}<1$ and the critical dimension is $k=\left\lfloor 1 / \alpha_{1}\right\rfloor=1$.

Remark 4.6. For the dynamic clique complex, the assumption (4.5) fails in a certain range of the parameter. Therefore, Theorem 4.2 does not claim the functional convergence in full generality, for the Euler characteristic and the Betti numbers in the critical dimension. On the other hand, Thoppe et al. [21] who only discuss this model, established tightness in full generality, and hence FCLT in the $J_{1}$-topology on $D[0, \infty)$. The reason for this discrepancy is the generality of our setup. In particular, in the dynamic clique complex, all Betti numbers except that in the critical dimension are known to vanish with a very high probability (see $[13,14]$ ), which makes it possible to obtain the required tightness in [21]. In the general multi-parameter simplicial complex, however, this is no longer necessarily the case, and the Betti number in the dimension greater than the critical one may not vanish; see Corollary 1.7 of Fowler [7]. To overcome the resulting difficulty, we have imposed an extra condition (4.5). We anticipate that the tightness holds without that extra condition; one way to avoid this is via very complicated fourth moment estimates for the Betti numbers based on the expression in Proposition A.6.

\section{Proof of the FCLT for the face counts}

In the sequel, we omit the subscript $n$ from all face count and Betti number notations. For example, we simply write $f_{j}(t), \beta_{j}(t)$ etc. Everywhere, $C$ denotes a generic positive constant, which is independent of $n$ but may vary between (or even within) the lines.

We start with proving the finite-dimensional convergence in Proposition 3.6. By the CramérWold device, it is enough to show that for all $0 \leq t_{1}<\cdots<t_{m}<\infty, a_{i} \in \mathbb{R}, i=1, \ldots, m$, $m \geq 1$,

$$
\frac{\sum_{i=1}^{m} a_{i}\left(f_{k}\left(t_{i}\right)-\mathbb{E}\left(f_{k}\right)\right)}{\sqrt{\operatorname{Var}\left(f_{k}\right)}} \Rightarrow \sum_{i=1}^{m} a_{i} Z_{k}\left(t_{i}\right) \text { in } \mathbb{R} .
$$

Clearly, it is enough to consider such choices of the coefficients for which the variance in the right hand side of (5.1) does not vanish, so fix such a set of coefficients. 
Let $J$ be the collection of $k$-faces or, equivalently, words of length $k+1$ in $[n]$. For $\mathbf{j} \in J$, let

$$
X_{\mathbf{j}}=\frac{\sum_{i=1}^{m} a_{i}\left(\xi_{\mathbf{j}}\left(t_{i}\right)-\mathbb{E}\left(\xi_{\mathbf{j}}\right)\right)}{\sqrt{\operatorname{Var}\left(\sum_{i=1}^{m} a_{i} f_{k}\left(t_{i}\right)\right)}}
$$

recall that $\xi_{\mathbf{j}}(t)$ is the indicator function that the $k$-face associated with the word $\mathbf{j}$ is "on" at time $t$. Finally, define

$$
W:=\sum_{\mathbf{j} \in J} X_{\mathbf{j}}=\frac{\sum_{i=1}^{m} a_{i}\left(f_{k}\left(t_{i}\right)-\mathbb{E}\left(f_{k}\right)\right)}{\sqrt{\operatorname{Var}\left(\sum_{i=1}^{m} a_{i} f_{k}\left(t_{i}\right)\right)}},
$$

so that $\mathbb{E}(W)=0$ and $\operatorname{Var}(W)=1$.

In the terminology of Barbour et al. [2] (see Equ. (2.7) therein), $\left(X_{\mathbf{j}}, \mathbf{j} \in J\right.$ ) constitutes a dissociated set of random variables. To see this, identify each $k$-face $\mathbf{j} \in J$ by the tuple $\mathbf{j}_{q} \equiv\left(j_{1}, \ldots, j_{\left(\begin{array}{c}k+1 \\ q+1\end{array}\right)}\right)$, where each $j_{i}$ corresponds to a $q$-face in $\mathbf{j}$. For example, when $k=3$ and $q=1$, identify the 3 -face $[1,2,3,4]$ by the tuple $([1,2],[1,3],[1,4], \ldots,[3,4])$. Then, for any sets $K, L \subset J_{q}:=\left\{\mathbf{j}_{q}: \mathbf{j} \in J\right\}$ such that

$$
\left|\bigcup_{\mathbf{j}_{q} \in K}\left\{j_{1}, \ldots, j_{\left(\begin{array}{c}
k+1 \\
q+1
\end{array}\right)}\right\} \cap \bigcup_{\mathbf{j}_{q}^{\prime} \in L}\left\{j_{1}^{\prime}, \ldots, j_{\left(\begin{array}{c}
k+1 \\
q+1
\end{array}\right)}^{\prime}\right\}\right|=\emptyset
$$

we have that $\left(X_{\mathbf{j}}: \mathbf{j}_{q} \in K\right)$ is independent of $\left(X_{\mathbf{j}}: \mathbf{j}_{q} \in L\right)$. This verifies the claim that $\left(X_{\mathbf{j}}: \mathbf{j} \in J\right)$ is a dissociated set of random variables. We can thus invoke the central limit theorem of Barbour et al. [2] for sums of dissociated random variables.

The approach is to estimate the $L_{1}$-Wasserstein metric between the distribution $\mathcal{L}_{W}$ of $W$ and the standard normal distribution, i.e.

$$
d_{1}\left(\mathcal{L}_{W}, \mathcal{L}_{Y}\right)=\sup _{\phi}|\mathbb{E}(\phi(W))-\mathbb{E}(\phi(Y))|,
$$

where $Y$ has the standard normal distribution and the supremum is taken over all $\phi: \mathbb{R} \rightarrow \mathbb{R}$ such that $\sup _{y_{1} \neq y_{2}}\left|\phi\left(y_{1}\right)-\phi\left(y_{2}\right)\right| /\left|y_{1}-y_{2}\right| \leq 1$. Assuming we have shown that $d_{1}\left(\mathcal{L}_{W}, \mathcal{L}_{Y}\right) \rightarrow$ 0 , we have $W \Rightarrow Y$ as $n \rightarrow \infty$. Furthermore, direct applications of Proposition 3.1 and (3.8) yield

$$
\frac{\operatorname{Var}\left(\sum_{i=1}^{m} a_{i} f_{k}\left(t_{i}\right)\right)}{\operatorname{Var}\left(f_{k}\right)} \rightarrow \operatorname{Var}\left(\sum_{i=1}^{m} a_{i} Z_{k}\left(t_{i}\right)\right), \quad n \rightarrow \infty .
$$

Therefore, $d_{1}\left(\mathcal{L}_{W}, \mathcal{L}_{Y}\right) \rightarrow 0$ would give us

$$
\frac{\sum_{i=1}^{m} a_{i}\left(f_{k}\left(t_{i}\right)-\mathbb{E}\left(f_{k}\right)\right)}{\sqrt{\operatorname{Var}\left(f_{k}\right)}} \Rightarrow\left\{\operatorname{Var}\left(\sum_{i=1}^{m} a_{i} Z_{k}\left(t_{i}\right)\right)\right\}^{1 / 2} Y \stackrel{d}{=} \sum_{i=1}^{m} a_{i} Z_{k}\left(t_{i}\right), \quad n \rightarrow \infty,
$$

as required.

It remains to actually show that $d_{1}\left(\mathcal{L}_{W}, \mathcal{L}_{Y}\right) \rightarrow 0$ as $n \rightarrow \infty$. Let $L_{\mathbf{j}}=\{\mathbf{k} \in J$ : $|\mathbf{k} \cap \mathbf{j}| \geq q+1\}$ be the dependency neighborhood of $\mathbf{j} \in J$, that is, a collection of simplices $\mathbf{k}$ having at least one $q$-face in common with $\mathbf{j}$. Then a slight reformulation of (3.4) in [2] and Proposition 3.1 shows that for a constant $C$ that may depend on the coefficients $a_{1}, \ldots, a_{m}$, 
but on nothing else,

$$
\begin{aligned}
d_{1}\left(\mathcal{L}_{W}, \mathcal{L}_{Y}\right) \leq C \sum_{\mathbf{j} \in J} \sum_{\mathbf{k} \in L_{\mathbf{j}}} \sum_{\mathbf{l} \in L_{\mathbf{j}}}\left\{\mathbb{E}\left(\left|X_{\mathbf{j}} X_{\mathbf{k}} X_{\mathbf{l}}\right|\right)+\mathbb{E}\left(\left|X_{\mathbf{j}} X_{\mathbf{k}}\right|\right) \mathbb{E}\left(\left|X_{\mathbf{l}}\right|\right)\right\} \\
\leq \frac{C}{n^{3 \tau_{k}(\boldsymbol{\alpha})-3 \tau_{q}(\boldsymbol{\alpha}) / 2}} \sum_{i_{1}, i_{2}, i_{3}=1}^{m} \sum_{\mathbf{j} \in J} \sum_{\mathbf{k} \in L_{\mathbf{j}}} \sum_{\mathbf{l} \in L_{\mathbf{j}}}\left\{\mathbb { E } \left[\left(\xi_{\mathbf{j}}\left(t_{i_{1}}\right)+\mathbb{E}\left(\xi_{\mathbf{j}}\right)\right)\left(\xi_{\mathbf{k}}\left(t_{i_{2}}\right)+\mathbb{E}\left(\xi_{\mathbf{k}}\right)\right)\right.\right. \\
\left.\times\left(\xi_{\mathbf{l}}\left(t_{i_{3}}\right)+\mathbb{E}\left(\xi_{\mathbf{l}}\right)\right)\right] \\
\left.+2 \mathbb{E}\left[\left(\xi_{\mathbf{j}}\left(t_{i_{1}}\right)+\mathbb{E}\left(\xi_{\mathbf{j}}\right)\right)\left(\xi_{\mathbf{k}}\left(t_{i_{2}}\right)+\mathbb{E}\left(\xi_{\mathbf{k}}\right)\right)\right] \mathbb{E}\left(\xi_{\mathbf{l}}\right)\right\} .
\end{aligned}
$$

For fixed $\mathbf{j} \in J, \mathbf{k} \in L_{\mathbf{j}}, \mathbf{l} \in L_{\mathbf{j}}$ denote

$$
\ell_{12}=|\mathbf{j} \cap \mathbf{k}|, \quad \ell_{13}=|\mathbf{j} \cap \mathbf{l}|, \quad \ell_{23}=|\mathbf{k} \cap \mathbf{l}|, \quad \ell_{123}=|\mathbf{j} \cap \mathbf{k} \cap \mathbf{l}| .
$$

Since $\mathbf{k}, \mathbf{l} \in L_{\mathbf{j}}$, it must be that $\ell_{12} \geq q+1$ and $\ell_{13} \geq q+1$, whereas $\ell_{23}$ and $\ell_{123}$ can be less than $q+1$. Given $\ell_{12}, \ell_{13}, \ell_{23}$, and $\ell_{123}$ as above, the expression between the braces in the right hand side of (5.2) can, up to a constant factor, be bounded by

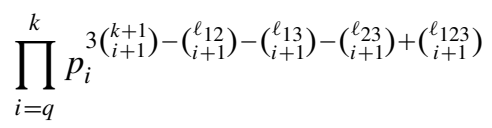

For example, for $0 \leq r \leq s \leq t<\infty$, by the inclusion-exclusion formula,

$$
\begin{aligned}
& \mathbb{E}\left(\xi_{\mathbf{j}}(r) \xi_{\mathbf{k}}(s) \xi_{\mathbf{l}}(t)\right) \\
= & \prod_{i=q}^{k} p_{i}^{3\left(\begin{array}{c}
k+1 \\
i+1
\end{array}\right)-\left(\begin{array}{c}
\ell_{12} \\
i+1
\end{array}\right)-\left(\begin{array}{c}
\ell_{13} \\
i+1
\end{array}\right)-\left(\begin{array}{c}
\ell_{23} \\
i+1
\end{array}\right)+\left(\begin{array}{c}
\ell_{123} \\
i+1
\end{array}\right)} \\
\times & \prod_{i=q}^{k} \mathbb{P}\left(\Delta_{i}(s)=1 \mid \Delta_{i}(r)=1\right)^{\left(\begin{array}{c}
\ell_{12} \\
i+1
\end{array}\right)-\left(\begin{array}{c}
\ell_{123} \\
i+1
\end{array}\right)} \prod_{i=q}^{k} \mathbb{P}\left(\Delta_{i}(t)=1 \mid \Delta_{i}(s)=1\right)^{\left(\begin{array}{c}
\ell_{2} 3 \\
i+1
\end{array}\right)-\left(\begin{array}{c}
\ell_{123} \\
i+1
\end{array}\right)} \\
\times & \prod_{i=q}^{k} \mathbb{P}\left(\Delta_{i}(t)=1 \mid \Delta_{i}(r)=1\right)^{\left(\begin{array}{c}
\ell_{13} \\
i+1
\end{array}\right)-\left(\begin{array}{l}
\ell_{123} \\
i+1
\end{array}\right)} \prod_{i=q}^{k} \mathbb{P}\left(\Delta_{i}(s)=\Delta_{i}(t)=1 \mid \Delta_{i}(r)=1\right)^{\left(\begin{array}{l}
\ell_{123} \\
i+1
\end{array}\right)} \\
\leq & \prod_{i=q}^{k} p_{i}^{3\left(\begin{array}{c}
k+1 \\
i+1
\end{array}\right)-\left(\begin{array}{c}
\ell_{12} \\
i+1
\end{array}\right)-\left(\begin{array}{l}
\ell_{13} \\
i+1
\end{array}\right)-\left(\begin{array}{c}
\ell_{23} \\
i+1
\end{array}\right)+\left(\begin{array}{c}
\ell_{123} \\
i+1
\end{array}\right)},
\end{aligned}
$$

and the terms of the other types can be bounded in a similar manner.

Furthermore, observe that for every $\ell_{12} \geq q+1, \ell_{13} \geq q+1, \ell_{23} \geq 0$, and $\ell_{123} \geq 0$, the number of the corresponding terms in (5.2) does not exceed a constant multiple of $n^{3(k+1)-\ell_{12}-\ell_{13}-\ell_{23}+\ell_{123}}$. Therefore,

$$
\begin{aligned}
d_{1}(W, Y) \leq & \frac{C}{n^{3 \tau_{k}(\boldsymbol{\alpha})-3 \tau_{q}(\boldsymbol{\alpha}) / 2}} \\
& \times \sum_{\ell_{12}=q+1}^{k+1} \sum_{\ell_{13}=q+1}^{k+1} \sum_{\ell_{23}=0}^{k+1} \sum_{\ell_{123}=0}^{\ell_{12} \wedge \ell_{13} \wedge \ell_{23}} \prod_{i=q}^{k} p_{i}^{3\left(\begin{array}{l}
k+1 \\
i+1
\end{array}\right)-\left(\begin{array}{l}
\ell_{12} \\
i+1
\end{array}\right)-\left(\begin{array}{l}
\ell_{13} \\
i+1
\end{array}\right)-\left(\begin{array}{l}
\ell_{23} \\
i+1
\end{array}\right)+\left(\begin{array}{c}
\ell_{123} \\
i+1
\end{array}\right)}
\end{aligned}
$$




$$
=C \sum_{\ell_{12}=q+1}^{k+1} \sum_{\ell_{13}=q+1}^{k+1} \sum_{\ell_{23}=0}^{k+1} \sum_{\ell_{123}=0}^{\ell_{12} \wedge \ell_{13} \wedge \ell_{23}} n^{3 \tau_{q}(\boldsymbol{\alpha}) / 2-\tau_{\ell_{12}-1}(\boldsymbol{\alpha})-\tau_{\ell_{13}-1}(\boldsymbol{\alpha})-\tau_{\ell_{23}-1}(\boldsymbol{\alpha})+\tau_{\ell_{123}-1}(\boldsymbol{\alpha})}
$$

$(a \wedge b=\min \{a, b\}$ for $a, b \in \mathbb{R})$. The latter sum is a finite sum, and each term in it does not exceed $C n^{-\tau_{q}(\boldsymbol{\alpha}) / 2}$ which can be seen by noticing that $\tau_{\ell_{23}-1}(\boldsymbol{\alpha})-\tau_{\ell_{123}-1}(\boldsymbol{\alpha})>0$ and setting $\ell_{12}=\ell_{13}=q+1$. Therefore, the sum goes to 0 as $n \rightarrow \infty$ and, hence, we have established the convergence of the finite-dimensional distributions in Proposition 3.6.

In order to prove tightness in the $J_{1}$-topology, we use Theorem 13.5 in [3]. By the stationarity of $f_{k}(t)$, it is sufficient to show that for every $T>0$, there exists $B>0$ such that

$$
\frac{\mathbb{E}\left[\left(f_{k}(t)-f_{k}(s)\right)^{2}\left(f_{k}(s)-f_{k}(r)\right)^{2}\right]}{\left(\operatorname{Var}\left(f_{k}\right)\right)^{2}} \leq B(t-r)^{1+\gamma}
$$

for all $0 \leq r \leq s \leq t \leq T, n \geq 1$, with $\gamma$ as in (2.9). By Proposition 3.1, we only need to show existence of $B$ such that

$$
\frac{\mathbb{E}\left[\left(f_{k}(t)-f_{k}(s)\right)^{2}\left(f_{k}(s)-f_{k}(r)\right)^{2}\right]}{n^{4 \tau_{k}(\boldsymbol{\alpha})-2 \tau_{q}(\boldsymbol{\alpha})}} \leq B(t-r)^{1+\gamma} .
$$

This will be established while proving tightness in the proof of Theorem 4.2.

\section{Proofs of the limit theorems for the Euler characteristic}

We start with the strong law of large numbers. As in the last section, $C$ denotes a generic positive constant, which is independent of $n$.

Proof of (4.1) in Theorem 4.1. Fix $0<T<\infty$ for the duration of the proof. We first check that for each $j \geq 0$,

$$
\sup _{0 \leq t \leq T} \frac{\left|f_{j}(t)-\mathbb{E}\left(f_{j}\right)\right|}{\mathbb{E}\left(f_{k}\right)} \rightarrow 0 \text { a.s. }
$$

If $j \in\{0, \ldots, q-1\}$, the left hand side is identically zero (see Remark 3.2). For $j \geq q$, by the Borel-Cantelli lemma, it suffices to show that for every $\epsilon>0$,

$$
\sum_{n=1}^{\infty} \mathbb{P}\left(\sup _{0 \leq t \leq T}\left|f_{j}(t)-\mathbb{E}\left(f_{j}\right)\right|>\epsilon \mathbb{E}\left(f_{k}\right)\right)<\infty,
$$

which will follow once we prove the following two statements:

$$
\begin{aligned}
& \sum_{n=1}^{\infty} \mathbb{P}\left(\sup _{0 \leq t \leq T} f_{j}(t)>\mathbb{E}\left(f_{j}\right)+\epsilon \mathbb{E}\left(f_{k}\right)\right)<\infty, \quad \text { and } \\
& \sum_{n=1}^{\infty} \mathbb{P}\left(\inf _{0 \leq t \leq T} f_{j}(t)<\mathbb{E}\left(f_{j}\right)-\epsilon \mathbb{E}\left(f_{k}\right)\right)<\infty .
\end{aligned}
$$

Choose a positive integer $m$ so large that

$$
\prod_{i=q}^{j}\left(1+\frac{\left(G_{i}\right)_{e}(T / m)}{1-G_{i}(T / m)}\right)^{\left(\begin{array}{c}
j+1 \\
i+1
\end{array}\right)}<1+\frac{\epsilon}{2}
$$


By stationarity,

$$
\mathbb{P}\left(\sup _{0 \leq t \leq T} f_{j}(t)>\mathbb{E}\left(f_{j}\right)+\epsilon \mathbb{E}\left(f_{k}\right)\right) \leq m \mathbb{P}\left(\sup _{0 \leq t \leq T / m} f_{j}(t)>\mathbb{E}\left(f_{j}\right)+\epsilon \mathbb{E}\left(f_{k}\right)\right) .
$$

We now construct a new static multi-parameter simplicial complex $X\left([n], \mathbf{p}^{(1)}\right)$ by setting $p_{i}^{(1)}=\mathbb{P}\left(\sup _{0 \leq t \leq T / m} \Delta_{i}(t)=1\right)$ for $i \geq 1$. If $f_{j}^{(1)}$ is the $j$-face count in this static complex, then, by a straightforward coupling argument,

$$
\sup _{0 \leq t \leq T / m} f_{j}(t) \stackrel{s t}{\leq} f_{j}^{(1)}
$$

Since by part (ii) of Lemma 2.4 and (6.4),

$$
\begin{aligned}
\mathbb{E}\left(f_{j}^{(1)}\right) & =\left(\begin{array}{c}
n \\
j+1
\end{array}\right) \prod_{i=q}^{j}\left(p_{i}^{(1)}\right)^{\left(\begin{array}{c}
j+1 \\
i+1
\end{array}\right)} \\
& \leq\left(\begin{array}{c}
n \\
j+1
\end{array}\right) \prod_{i=q}^{j} p_{i}^{\left(\begin{array}{c}
j+1 \\
i+1
\end{array}\right)} \prod_{i=q}^{j}\left(1+\frac{\left(G_{i}\right)_{e}(T / m)}{1-G_{i}(T / m)}\right)^{\left(\begin{array}{c}
j+1 \\
i+1
\end{array}\right)} \leq\left(1+\frac{\epsilon}{2}\right) \mathbb{E}\left(f_{j}\right),
\end{aligned}
$$

we conclude that

$$
\begin{aligned}
\mathbb{P}\left(\sup _{0 \leq t \leq T / m} f_{j}(t)>\mathbb{E}\left(f_{j}\right)+\epsilon \mathbb{E}\left(f_{k}\right)\right) & \leq \mathbb{P}\left(f_{j}^{(1)}-\mathbb{E}\left(f_{j}^{(1)}\right)>\mathbb{E}\left(f_{j}\right)+\epsilon \mathbb{E}\left(f_{k}\right)-\mathbb{E}\left(f_{j}^{(1)}\right)\right) \\
& \leq \mathbb{P}\left(f_{j}^{(1)}-\mathbb{E}\left(f_{j}^{(1)}\right)>\epsilon \mathbb{E}\left(f_{k}\right)-\frac{\epsilon}{2} \mathbb{E}\left(f_{j}\right)\right) .
\end{aligned}
$$

As $\mathbb{E}\left(f_{j}\right) / \mathbb{E}\left(f_{k}\right) \rightarrow 0, n \rightarrow \infty$ for $j \neq k$, it holds that, for sufficiently large $n$,

$$
\begin{aligned}
\mathbb{P}\left(f_{j}^{(1)}-\mathbb{E}\left(f_{j}^{(1)}\right)>\epsilon \mathbb{E}\left(f_{k}\right)-\frac{\epsilon}{2} \mathbb{E}\left(f_{j}\right)\right) & \leq \mathbb{P}\left(\left|f_{j}^{(1)}-\mathbb{E}\left(f_{j}^{(1)}\right)\right|>\frac{\epsilon}{2} \mathbb{E}\left(f_{k}\right)\right) \\
& \leq \frac{4}{\epsilon^{2}} \frac{\operatorname{Var}\left(f_{j}^{(1)}\right)}{\left(\mathbb{E}\left(f_{k}\right)\right)^{2}} \leq C \frac{\operatorname{Var}\left(f_{j}^{(1)}\right)}{n^{2 \tau_{k}(\boldsymbol{\alpha})}},
\end{aligned}
$$

where the last inequality comes from Proposition 3.1. Further, since each $p_{i}^{(1)}$ is asymptotically bounded by $p_{i}$ times a positive constant for $i=q, \ldots, j$, the argument of the above proposition shows that for large enough $n$,

$$
\operatorname{Var}\left(f_{j}^{(1)}\right) \leq C_{j}^{(1)} n^{2 \tau_{j}(\boldsymbol{\alpha})-\tau_{q}(\boldsymbol{\alpha})} \vee C_{j}^{(2)} n^{\tau_{j}(\boldsymbol{\alpha})}
$$

for some finite positive constants $C_{j}^{(1)}$ and $C_{j}^{(2)}$. Hence,

$$
\begin{aligned}
\mathbb{P}\left(\sup _{0 \leq t \leq T / m} f_{j}(t)>\mathbb{E}\left(f_{j}\right)+\epsilon \mathbb{E}\left(f_{k}\right)\right) & \leq C \frac{n^{2 \tau_{j}(\boldsymbol{\alpha})-\tau_{q}(\boldsymbol{\alpha})} \vee n^{\tau_{j}(\boldsymbol{\alpha})}}{n^{2 \tau_{k}(\boldsymbol{\alpha})}} \\
& \leq C n^{-\tau_{q}(\boldsymbol{\alpha})} \leq C n^{-\tau_{1}(\boldsymbol{\alpha})}=C n^{-\left(2-\alpha_{1}\right)} .
\end{aligned}
$$

As $\alpha_{1}=\psi_{1}(\boldsymbol{\alpha}) \leq \psi_{k}(\boldsymbol{\alpha})<1$, we get $\sum_{n=1}^{\infty} n^{-\left(2-\alpha_{1}\right)}<\infty$, and so (6.2) holds.

We now turn our attention to (6.3). The stationarity of $f_{j}(t)$ implies that

$$
\mathbb{P}\left(\inf _{0 \leq t \leq T} f_{j}(t)<\mathbb{E}\left(f_{j}\right)-\epsilon \mathbb{E}\left(f_{k}\right)\right) \leq m \mathbb{P}\left(\inf _{0 \leq t \leq T / m} f_{j}(t)<\mathbb{E}\left(f_{j}\right)-\epsilon \mathbb{E}\left(f_{k}\right)\right),
$$

where this time $m$ is chosen so that

$$
\prod_{i=q}^{j}\left(1-\left(G_{i}\right)_{e}(T / m)\right)^{\left(\begin{array}{c}
j+1 \\
i+1
\end{array}\right)}>1-\frac{\epsilon}{2} .
$$


Once again, we construct a new static multi-parameter simplicial complex $X\left([n], \mathbf{p}^{(2)}\right)$ by setting this time $p_{i}^{(2)}=\mathbb{P}\left(\inf _{0 \leq t \leq T / m} \Delta_{i}(t)=1\right)$ for $i \geq 1$. If $f_{j}^{(2)}$ is the $j$-face count in this static complex, then, $f_{j}^{(2)} \stackrel{s t}{\leq} \inf _{0 \leq t \leq T / m} f_{j}(t)$. Notice that for $i \geq q$,

$$
p_{i}^{(2)} \geq \mathbb{P}\left(\Delta_{i}(0)=1, D^{(i)} \geq T / m\right)=p_{i}\left(1-\left(G_{i}\right)_{e}(T / m)\right),
$$

so by the choice of $m$,

$$
\mathbb{E}\left(f_{j}^{(2)}\right) \geq\left(1-\frac{\epsilon}{2}\right) \mathbb{E}\left(f_{j}\right) .
$$

Proceeding as above we conclude that, for sufficiently large $n$,

$$
\mathbb{P}\left(\inf _{0 \leq t \leq T / m} f_{j}(t)<\mathbb{E}\left(f_{j}\right)-\epsilon \mathbb{E}\left(f_{k}\right)\right) \leq C \frac{\operatorname{Var}\left(f_{j}^{(2)}\right)}{n^{2 \tau_{k}(\boldsymbol{\alpha})}} .
$$

Noting that $p_{i}^{(2)} \leq p_{i}^{(1)}$, the same logic as above tells that

$$
\operatorname{Var}\left(f_{j}^{(2)}\right) \leq C_{j}^{(1)} n^{2 \tau_{j}(\boldsymbol{\alpha})-\tau_{q}(\boldsymbol{\alpha})} \vee C_{j}^{(2)} n^{\tau_{j}(\boldsymbol{\alpha})}
$$

for some finite positive constants $C_{j}^{(1)}, C_{j}^{(2)}$, and (6.3) follows in the same way as (6.2) did.

The next step is to show that as $n \rightarrow \infty$,

$$
\sup _{0 \leq t \leq T} \frac{\left|\chi_{n}(t)-\mathbb{E}\left(\chi_{n}\right)\right|}{\mathbb{E}\left(f_{k}\right)} \rightarrow 0 \text { a.s., }
$$

and by stationarity it is enough to prove that

$$
\sup _{0 \leq t \leq T / m} \frac{\left|\chi_{n}(t)-\mathbb{E}\left(\chi_{n}\right)\right|}{\mathbb{E}\left(f_{k}\right)} \rightarrow 0 \text { a.s. }
$$

for an integer $m$ large enough so that $T / m \leq a / 4$; the constant $a$ is given in the assumption (2.3). It is not difficult to see that the choice of $m$ implies $\left(G_{i}\right)_{e}(T / m) \leq 1 / 2$. Combining this with part (ii) of Lemma 2.4 and recalling that $p_{i}^{(1)}=\mathbb{P}\left(\sup _{0 \leq t \leq T / m} \bar{\Delta}_{i}(t)=1\right)$, we get $p_{i}^{(1)} \leq p_{i}\left(2-p_{i}\right)$. It is now elementary to check that there is a function $h:[0, \infty] \rightarrow[0, \infty]$ with $h(0)=0, h(\infty)=\infty$, and $h(\alpha) \in(0, \infty)$ for $0<\alpha<\infty$, such that

$$
p_{i}^{(1)} \leq p_{i}\left(2-p_{i}\right) \leq n^{-h\left(\alpha_{i}\right)} \text { if } p_{i}=n^{-\alpha_{i}}, i \geq 1 \text {; }
$$

(for example, one may take $h(\alpha)=\alpha-\log \left(2-2^{-\alpha}\right) / \log 2$ ). Define now $\tilde{\boldsymbol{\alpha}}$ by $\tilde{\alpha}_{i}=h\left(\alpha_{i}\right)$, $i=1,2, \ldots$. Then, $M(\tilde{\boldsymbol{\alpha}})$ defined by (3.7) is finite, and we use (3.2) to bound

$$
\begin{aligned}
\sup _{0 \leq t \leq T / m} \frac{\left|\chi_{n}(t)-\mathbb{E}\left(\chi_{n}\right)\right|}{\mathbb{E}\left(f_{k}\right)} \leq \sum_{j=0}^{M(\tilde{\alpha})-1} & \sup _{0 \leq t \leq T / m} \frac{\left|f_{j}(t)-\mathbb{E}\left(f_{j}\right)\right|}{\mathbb{E}\left(f_{k}\right)} \\
& +\sum_{j=M(\tilde{\alpha})}^{n-1} \sup _{0 \leq t \leq T / m} \frac{\left|f_{j}(t)-\mathbb{E}\left(f_{j}\right)\right|}{\mathbb{E}\left(f_{k}\right)} .
\end{aligned}
$$

By (6.1), the first sum in the right hand side almost surely goes to 0 as $n \rightarrow \infty$. For the second sum, we again use the Borel-Cantelli lemma by initially showing that, for every $\epsilon>0$,

$$
\sum_{n=M(\tilde{\boldsymbol{\alpha}})+1}^{\infty} \mathbb{P}\left(\sum_{j=M(\tilde{\boldsymbol{\alpha}})}^{n-1} \sup _{0 \leq t \leq T / m}\left|f_{j}(t)-\mathbb{E}\left(f_{j}\right)\right|>\epsilon \mathbb{E}\left(f_{k}\right)\right)<\infty .
$$


Using Markov's inequality and recalling our notation for the face counts in the static multiparameter simplicial complex $X\left([n], \mathbf{p}^{(1)}\right)$, we bound the above sum by

$$
\frac{2}{\epsilon} \sum_{n=M(\tilde{\boldsymbol{\alpha}})+1}^{\infty} \frac{1}{\mathbb{E}\left(f_{k}\right)} \sum_{j=M(\tilde{\boldsymbol{\alpha}})}^{n-1} \mathbb{E}\left(\sup _{0 \leq t \leq T / m} f_{j}(t)\right) \leq \frac{2 C}{\epsilon} \sum_{n=1}^{\infty} \frac{1}{n^{\tau_{1}(\boldsymbol{\alpha})}} \sum_{j=M(\tilde{\boldsymbol{\alpha}})}^{\infty} \mathbb{E}\left(f_{j}^{(1)}\right)<\infty
$$

since $\sum_{n=1}^{\infty} n^{-\tau_{1}(\boldsymbol{\alpha})}<\infty$ and $\sum_{j=M(\tilde{\boldsymbol{\alpha}})}^{\infty} \mathbb{E}\left(f_{j}^{(1)}\right) \rightarrow 0$ as $n \rightarrow \infty$ by Corollary 3.4. We have now obtained (6.7) and, hence, also (6.6).

Finally, we can use (3.2) to write

$$
\frac{\mathbb{E}\left(\chi_{n}\right)}{\mathbb{E}\left(f_{k}\right)}=(-1)^{k}+\frac{\sum_{j=0, j \neq k}^{n-1}(-1)^{j} \mathbb{E}\left(f_{j}\right)}{\mathbb{E}\left(f_{k}\right)} .
$$

With $M(\boldsymbol{\alpha})$ defined by (3.7),

$$
\left|\frac{\sum_{j=0, j \neq k}^{n-1}(-1)^{j} \mathbb{E}\left(f_{j}\right)}{\mathbb{E}\left(f_{k}\right)}\right| \leq \sum_{j=0, j \neq k}^{M(\boldsymbol{\alpha})-1} \frac{\mathbb{E}\left(f_{j}\right)}{\mathbb{E}\left(f_{k}\right)}+C \sum_{j=M(\boldsymbol{\alpha})}^{\infty} \mathbb{E}\left(f_{j}\right) \rightarrow 0, \quad n \rightarrow \infty
$$

by Proposition 3.1 and Corollary 3.4. Hence $\mathbb{E}\left(\chi_{n}\right) / \mathbb{E}\left(f_{k}\right) \rightarrow(-1)^{k}$, and (4.1) follows.

We now prove the functional central limit theorem for the Euler characteristic.

Proof of (4.3) in Theorem 4.2. Note, first of all, that for every $M \geq k+1$ the truncated Euler characteristic

$$
\chi_{n}^{(M)}(t)=\sum_{j=0}^{M-1}(-1)^{j} f_{j}(t)
$$

satisfies, in terms of convergence of the finite-dimensional distributions,

$$
\left(\frac{\chi_{n}^{(M)}(t)-\mathbb{E}\left(\chi_{n}^{(M)}\right)}{\sqrt{\operatorname{Var}\left(f_{k, n}\right)}}, t \geq 0\right) \Rightarrow\left(Z_{k}(t), t \geq 0\right) .
$$

This follows from finite-dimensional convergence in Proposition 3.6 and the fact that by (3.6) and Chebyshev's inequality,

$$
\frac{f_{j}(t)-\mathbb{E}\left(f_{j}\right)}{\sqrt{\operatorname{Var}\left(f_{k}\right)}} \stackrel{p}{\rightarrow} 0, \quad n \rightarrow \infty,
$$

for each $j \neq k$.

Choosing now $M=M(\boldsymbol{\alpha})$ defined by (3.7), we have by Corollary 3.4 that

$$
\mathbb{P}\left(\left|\frac{\chi_{n}(t)-\mathbb{E}\left(\chi_{n}\right)}{\sqrt{\operatorname{Var}\left(f_{k}\right)}}-\frac{\chi_{n}^{(M(\boldsymbol{\alpha}))}(t)-\mathbb{E}\left(\chi_{n}^{(M(\boldsymbol{\alpha}))}\right)}{\sqrt{\operatorname{Var}\left(f_{k}\right)}}\right|>\epsilon\right) \leq \frac{2}{\epsilon \sqrt{\operatorname{Var}\left(f_{k}\right)}} \sum_{j=M(\boldsymbol{\alpha})}^{\infty} \mathbb{E}\left(f_{j}\right) \rightarrow 0
$$

as $n \rightarrow \infty$ for any $\epsilon>0$. Therefore,

$$
\frac{\chi_{n}(t)-\mathbb{E}\left(\chi_{n}\right)}{\sqrt{\operatorname{Var}\left(f_{k}\right)}}-\frac{\chi_{n}^{(M(\alpha))}(t)-\mathbb{E}\left(\chi_{n}^{(M(\alpha))}\right)}{\sqrt{\operatorname{Var}\left(f_{k}\right)}} \stackrel{p}{\rightarrow} 0,
$$

so we have established (4.3) in terms of convergence of the finite-dimensional distributions. 
Assuming (2.9) and (4.5), we now establish tightness in the Skorohod $J_{1}$-topology. Denote

$$
M_{1}(\boldsymbol{\alpha})=\min \left\{i>k: \tau_{i}(\boldsymbol{\alpha})<\tau_{q}(\boldsymbol{\alpha})\right\} .
$$

Fix $T>0$ and choose $m$ so that $T / m \leq a / 4$, where $a$ is the constant from (2.3). Recall once again the notation $p_{i}^{(1)}=\mathbb{P}\left(\sup _{0 \leq t \leq T / m} \Delta_{i}(t)=1\right), i \geq 1$, so that $p_{i}^{(1)} \leq p_{i}\left(2-p_{i}\right) \leq n^{-\tilde{\alpha}_{i}}$, where $\tilde{\boldsymbol{\alpha}}=\left(\tilde{\alpha}_{1}, \tilde{\alpha}_{2}, \ldots\right)$ is as defined below (6.8). Note that $M_{1}(\boldsymbol{\alpha}) \leq M(\boldsymbol{\alpha}) \leq M(\tilde{\boldsymbol{\alpha}})<\infty$, where $M(\boldsymbol{\alpha})$ and $M(\tilde{\boldsymbol{\alpha}})$ are as defined in (3.7). Recall also that for $j \geq q, f_{j}^{(1)}$ is the $j$-face counts in $X\left([n], \mathbf{p}^{(1)}\right)$, such that $f_{j}^{(1)} \stackrel{s d}{\geq} \sup _{0 \leq t \leq T / m} f_{j}(t)$. Write

$$
\chi_{n}(t)=\sum_{j=0}^{M_{1}(\boldsymbol{\alpha})-1}(-1)^{j} f_{j}(t)+\sum_{j=M_{1}(\boldsymbol{\alpha})}^{n-1}(-1)^{j} f_{j}(t)=: \chi_{n}^{(1)}(t)+\chi_{n}^{(2)}(t), 0 \leq t \leq T .
$$

We start with proving that, as $n \rightarrow \infty$,

$$
\frac{\sup _{0 \leq t \leq T}\left|\chi_{n}^{(2)}(t)-\mathbb{E}\left(\chi_{n}^{(2)}\right)\right|}{\sqrt{\operatorname{Var}\left(f_{k}\right)}} \stackrel{p}{\rightarrow} 0 .
$$

By stationarity, it suffices to show that

$$
\frac{\sup _{0 \leq t \leq T / m}\left|\chi_{n}^{(2)}(t)-\mathbb{E}\left(\chi_{n}^{(2)}\right)\right|}{\sqrt{\operatorname{Var}\left(f_{k}\right)}} \stackrel{p}{\rightarrow} 0 .
$$

Let $\epsilon>0$ be arbitrary. Then, by Markov's inequality, for all sufficiently large $n$,

$$
\begin{aligned}
& \mathbb{P}\left(\sup _{0 \leq t \leq T / m}\left|\chi_{n}^{(2)}(t)-\mathbb{E}\left(\chi_{n}^{(2)}\right)\right|>\epsilon \sqrt{\operatorname{Var}\left(f_{k}\right)}\right) \\
& \leq \frac{2}{\epsilon \sqrt{\operatorname{Var}\left(f_{k}\right)}} \sum_{j=M_{1}(\boldsymbol{\alpha})}^{n-1} \mathbb{E}\left[\sup _{0 \leq t \leq T / m} f_{j}(t)\right] \\
& \leq \frac{2}{\epsilon \sqrt{\operatorname{Var}\left(f_{k}\right)}} \sum_{j=M_{1}(\boldsymbol{\alpha})}^{n-1} \mathbb{E}\left(f_{j}^{(1)}\right) \\
& \leq \frac{2}{\epsilon} \sum_{j=M_{1}(\boldsymbol{\alpha})}^{M(\tilde{\boldsymbol{\alpha}})-1} \frac{\prod_{i=q}^{j} 2^{\left(\begin{array}{c}
j+1 \\
i+1
\end{array}\right) \mathbb{E}\left(f_{j}\right)}}{\sqrt{\operatorname{Var}\left(f_{k}\right)}}+\frac{2}{\epsilon} \sum_{j=M(\tilde{\boldsymbol{\alpha}})}^{\infty} \mathbb{E}\left(f_{j}^{(1)}\right),
\end{aligned}
$$

where the last inequality is due to Proposition 3.1, together with the fact that $p_{i}^{(1)} \leq 2 p_{i}$. The second term vanishes because $\sum_{j=M(\tilde{\alpha})}^{\infty} \mathbb{E}\left(f_{j}^{(1)}\right) \rightarrow 0$, as $n \rightarrow \infty$, by Corollary 3.4. On the other hand, the first vanishes since, by (4.5),

$$
\mathbb{E}\left(f_{j}\right) \leq n^{\tau_{j}(\boldsymbol{\alpha})} \leq n^{\tau_{k+1}(\boldsymbol{\alpha})}=o\left(n^{\tau_{k}(\boldsymbol{\alpha})-\tau_{q}(\boldsymbol{\alpha}) / 2}\right)=o\left(\sqrt{\operatorname{Var}\left(f_{k}\right)}\right), \quad n \rightarrow \infty .
$$

Now (6.10) follows as desired, and so it remains to prove the tightness of $\left(\chi_{n}^{(1)}(t), 0 \leq t \leq T\right)$. For this, it is enough to show the existence of $B \in(0, \infty)$ such that

$$
\frac{\mathbb{E}\left[\left(\chi_{n}^{(1)}(t)-\chi_{n}^{(1)}(s)\right)^{2}\left(\chi_{n}^{(1)}(s)-\chi_{n}^{(1)}(r)\right)^{2}\right]}{n^{4 \tau_{k}(\alpha)-2 \tau_{q}(\alpha)}} \leq B(t-r)^{1+\gamma}
$$

for all $0 \leq r \leq s \leq t \leq T$ and $n \geq 1$. In the course of the proof, we will also establish (5.3) which is needed for the tightness in Proposition 3.6. 
We begin by setting up the notation. For $q+1 \leq j_{1}, j_{2}<M_{1}(\boldsymbol{\alpha})$ and $0 \leq r \leq s \leq t \leq T$, denote

$$
F_{j_{1}, j_{2}}(t, s, r):=\mathbb{E}\left[\left(f_{j_{1}}(t)-f_{j_{1}}(s)\right)^{2}\left(f_{j_{2}}(s)-f_{j_{2}}(r)\right)^{2}\right] .
$$

Consider a potential subcomplex $\bar{\sigma}$ in $[n]$ consisting of the 4 simplices $\sigma_{1}, \sigma_{2}, \sigma_{3}, \sigma_{4}$ and their faces, with $\left|\sigma_{1}\right|=\left|\sigma_{2}\right|=j_{1}+1,\left|\sigma_{3}\right|=\left|\sigma_{4}\right|=j_{2}+1$, and let

$$
\begin{aligned}
& a_{i j}=\left|\sigma_{i} \cap \sigma_{j}\right|, \quad 1 \leq i<j \leq 4, \quad a_{i j k}=\left|\sigma_{i} \cap \sigma_{j} \cap \sigma_{k}\right|, \quad 1 \leq i<j<k \leq 4, \\
& a_{1234}=\left|\sigma_{1} \cap \sigma_{2} \cap \sigma_{3} \cap \sigma_{4}\right| .
\end{aligned}
$$

The number of $i$-faces in $\bar{\sigma}$ is

$$
\begin{aligned}
\operatorname{comb}_{i}(\bar{\sigma}):= & 2\left(\begin{array}{c}
j_{1}+1 \\
i+1
\end{array}\right)+2\left(\begin{array}{c}
j_{2}+1 \\
i+1
\end{array}\right)-\left(\begin{array}{c}
a_{12} \\
i+1
\end{array}\right)-\left(\begin{array}{c}
a_{13} \\
i+1
\end{array}\right)-\left(\begin{array}{c}
a_{14} \\
i+1
\end{array}\right)-\left(\begin{array}{c}
a_{23} \\
i+1
\end{array}\right) \\
& -\left(\begin{array}{c}
a_{24} \\
i+1
\end{array}\right)-\left(\begin{array}{c}
a_{34} \\
i+1
\end{array}\right)+\left(\begin{array}{c}
a_{123} \\
i+1
\end{array}\right)+\left(\begin{array}{c}
a_{124} \\
i+1
\end{array}\right) \\
& +\left(\begin{array}{c}
a_{134} \\
i+1
\end{array}\right)+\left(\begin{array}{c}
a_{234} \\
i+1
\end{array}\right)-\left(\begin{array}{c}
a_{1234} \\
i+1
\end{array}\right) ;
\end{aligned}
$$

it depends only on $j_{1}, j_{2}$, and $\mathbf{a}=\left(a_{12}, \ldots, a_{1234}\right)$. We let

$$
\begin{aligned}
\Psi(\mathbf{a}, \boldsymbol{\alpha}):= & \tau_{a_{12}-1}(\boldsymbol{\alpha})+\tau_{a_{13}-1}(\boldsymbol{\alpha})+\tau_{a_{14-1}}(\boldsymbol{\alpha})+\tau_{a_{23}-1}(\boldsymbol{\alpha})+\tau_{a_{24}-1}(\boldsymbol{\alpha})+\tau_{a_{34}-1}(\boldsymbol{\alpha}) \\
& -\tau_{a_{123}-1}(\boldsymbol{\alpha})-\tau_{a_{124}-1}(\boldsymbol{\alpha})-\tau_{a_{134}-1}(\boldsymbol{\alpha})-\tau_{a_{234}-1}(\boldsymbol{\alpha})+\tau_{a_{1234}-1}(\boldsymbol{\alpha})
\end{aligned}
$$

(with $\tau_{-1}(\boldsymbol{\alpha}) \equiv 0$ ). By independence,

$$
\begin{aligned}
F_{j_{1}, j_{2}}(t, s, r)= & \sum_{\bar{\sigma} \subset \Xi\left(j_{1}, j_{2}\right)} \mathbb{E}\left[\left(\xi_{\sigma_{1}}(t)-\xi_{\sigma_{1}}(s)\right)\left(\xi_{\sigma_{2}}(t)-\xi_{\sigma_{2}}(s)\right)\left(\xi_{\sigma_{3}}(s)-\xi_{\sigma_{3}}(r)\right)\right. \\
& \left.\times\left(\xi_{\sigma_{4}}(s)-\xi_{\sigma_{4}}(r)\right)\right] \\
= & : \sum_{\bar{\sigma} \subset \Xi\left(j_{1}, j_{2}\right)} \mathbb{E}[g(t, s, r ; \bar{\sigma})],
\end{aligned}
$$

with the summation restricted to the set

$$
\begin{aligned}
& \Xi\left(j_{1}, j_{2}\right)=\left\{\bar{\sigma}=\left(\sigma_{1}, \ldots, \sigma_{4}\right):\left|\sigma_{1}\right|=\left|\sigma_{2}\right|=j_{1}+1,\left|\sigma_{3}\right|=\left|\sigma_{4}\right|=j_{2}+1,\right. \\
& \left.\quad \text { and }\left(\sigma_{1}, \ldots, \sigma_{4}\right) \text { satisfies at least one of the conditions in (6.16) below }\right\} \text { : } \\
& \text { (i) } a_{12} \geq q+1, a_{34} \geq q+1, \text { (ii) } a_{13} \geq q+1, a_{24} \geq q+1, \\
& \text { (iii) } a_{14} \geq q+1, a_{23} \geq q+1, \text { (iv) } a_{12} \geq q+1, a_{13} \geq q+1, a_{14} \geq q+1, \\
& \text { (v) } a_{12} \geq q+1, a_{23} \geq q+1, a_{24} \geq q+1 \text {, (vi) } a_{13} \geq q+1, a_{23} \geq q+1, a_{34} \geq q+1, \\
& \text { (vii) } a_{14} \geq q+1, a_{24} \geq q+1, a_{34} \geq q+1 .
\end{aligned}
$$

Indeed, if none of the conditions in (6.16) holds, then the corresponding term in (6.15) vanishes by independence and stationarity. 
Our goal is to bound the expectation $\mathbb{E}[g(t, s, r ; \bar{\sigma})]$ in $(6.15)$. Note that $g(t, s, r ; \bar{\sigma}) \in$ $\{-1,0,+1\}$. Hence, for $g(t, s, r ; \bar{\sigma})$ not to vanish, every $i$-face of the simplex $\sigma_{1}$ must exist either at time $s$ or at time $t, i=q, \ldots, j_{1}$, and the same is true for the simplex $\sigma_{2}$. Similarly, every $i$-face of the simplex $\sigma_{3}$ must exist either at time $r$ or at time $s, i=q, \ldots, j_{2}$, and the same is true for the simplex $\sigma_{4}$. The probability that this happens is bounded from above by

$$
16 \prod_{i=q}^{j_{1} \vee j_{2}} p_{i}^{\operatorname{comb}_{i}(\bar{\sigma})}
$$

where we take into account only the first (smallest) time a face exists if it is required to exist multiple times. Additionally, at least one face of the complex spanned by the simplices $\sigma_{1}, \sigma_{2}$ must switch from existence to non-existence, or vice versa, between times $s$ and $t$, and at least one face of the complex spanned by the simplices $\sigma_{3}, \sigma_{4}$ must switch from existence to non-existence, or vice versa, between times $r$ and $s$. This may be the same face or two different faces. Let us denote the corresponding (non-disjoint) events by $A_{1}$ and $A_{2}$. Consider the event $A_{1}$ first. The number of possible faces that can change their status does not exceed the total number of faces in $\bar{\sigma}$, which is, in turn, bounded by $2^{2\left(j_{1}+j_{2}\right)}$. For such an $i$-face the probability $p_{i}$ in (6.17) will be replaced by one by of following two probabilities:

$$
\mathbb{P}\left(\Delta_{i}(r)=0, \Delta_{i}(s)=1, \Delta_{i}(t)=0\right)
$$

and

$$
\mathbb{P}\left(\Delta_{i}(r)=1, \Delta_{i}(s)=0, \Delta_{i}(t)=1\right),
$$

both of which are bounded by $(2 c / a) p_{i}(t-r)^{1+\gamma}$ by Lemma 2.5. Therefore,

$$
\mathbb{P}\left(A_{1}\right) \leq C 2^{2\left(j_{1}+j_{2}\right)}(t-r)^{1+\gamma} \prod_{i=q}^{j_{1} \vee j_{2}} p_{i}^{\operatorname{comb}_{i}(\bar{\sigma})} .
$$

Considering the event $A_{2}$ now, we see that the number of possible pairs of faces that can change their status does not exceed $2^{4\left(j_{1}+j_{2}\right)}$. For each such a pair of an $i_{1}$-face and an $i_{2}$-face, the product $p_{i_{1}} p_{i_{2}}$ in (6.17) will be, up to renaming, replaced by

$$
\mathbb{P}\left(\Delta_{i_{1}}(r)=1, \Delta_{i_{1}}(s)=0\right) \mathbb{P}\left(\Delta_{i_{2}}(s)=1, \Delta_{i_{2}}(t)=0\right),
$$

or similar expressions obtained by flipping 1s and 0s. By Lemma 2.4, any such expression is bounded by

$$
p_{i_{1}} p_{i_{2}}\left(\frac{2}{a}\right)^{2}(t-r)^{2} .
$$

Since $\gamma \leq 1$, we conclude that

$$
\mathbb{P}\left(A_{2}\right) \leq C 2^{4\left(j_{1}+j_{2}\right)}(t-r)^{1+\gamma} \prod_{i=q}^{j_{1} \vee j_{2}} p_{i}^{\mathrm{comb}_{i}(\bar{\sigma})},
$$

and so

$$
\mathbb{E}[|g(t, s, r ; \bar{\sigma})|] \leq C 2^{4\left(j_{1}+j_{2}\right)}(t-r)^{1+\gamma} \prod_{i=q}^{j_{1} \vee j_{2}} p_{i}^{\operatorname{comb}_{i}(\bar{\sigma})}
$$


Substituting this back into (6.15), we obtain

$$
\begin{aligned}
F_{j_{1}, j_{2}}(t, s, r) \leq C 2^{4\left(j_{1}+j_{2}\right)}(t-r)^{1+\gamma} \sum_{\bar{\sigma} \in \Xi\left(j_{1}, j_{2}\right)} \prod_{i=q}^{j_{1} \vee j_{2}} p_{i}^{\operatorname{comb}_{i}(\bar{\sigma})} \\
=C 2^{4\left(j_{1}+j_{2}\right)}(t-r)^{1+\gamma} \sum_{\mathbf{a} \in \mathcal{A}} \sum_{\bar{\sigma} \in \Xi\left(j_{1}, j_{2}\right)} \mathbb{1}\left\{\left|\sigma_{1} \cap \sigma_{2}\right|=a_{12},\left|\sigma_{1} \cap \sigma_{3}\right|=a_{13}, \ldots,\right. \\
\left.\left|\sigma_{1} \cap \sigma_{2} \cap \sigma_{3} \cap \sigma_{4}\right|=a_{1234}\right\} \prod_{i=q}^{j_{1} \vee j_{2}} p_{i}^{\operatorname{comb}_{i}(\bar{\sigma})},
\end{aligned}
$$

where $\mathcal{A}$ is the collection of $\mathbf{a}=\left(a_{12}, \ldots, a_{1234}\right)$ satisfying at least one of the conditions in (6.16). Note that $\operatorname{comb}_{i}(\bar{\sigma})$ depends only on $\mathbf{a}$, and for any $\mathbf{a}$,

$$
\sum_{\bar{\sigma} \in \Xi\left(j_{1}, j_{2}\right)} \mathbb{1}\left\{\left|\sigma_{1} \cap \sigma_{2}\right|=a_{12},\left|\sigma_{1} \cap \sigma_{3}\right|=a_{13}, \ldots,\left|\sigma_{1} \cap \sigma_{2} \cap \sigma_{3} \cap \sigma_{4}\right|=a_{1234}\right\} \leq n^{\operatorname{comb}_{0}(\bar{\sigma})} .
$$

Since

$$
n^{\operatorname{comb}_{0}(\bar{\sigma})} \prod_{i=q}^{j_{1} \vee j_{2}} p_{i}^{\operatorname{comb}_{i}(\bar{\sigma})}=n^{2\left(\tau_{j_{1}}(\boldsymbol{\alpha})+\tau_{j_{2}}(\boldsymbol{\alpha})\right)-\Psi(\mathbf{a}, \boldsymbol{\alpha})}
$$

with $\Psi(\mathbf{a}, \boldsymbol{\alpha})$ given in (6.14), we obtain

$$
F_{j_{1}, j_{2}}(t, s, r) \leq C 2^{4\left(j_{1}+j_{2}\right)}(t-r)^{1+\gamma} \sum_{\mathbf{a} \in \mathcal{A}} n^{2\left(\tau_{j_{1}}(\boldsymbol{\alpha})+\tau_{j_{2}}(\boldsymbol{\alpha})\right)-\Psi(\mathbf{a}, \boldsymbol{\alpha})} .
$$

We proceed with the following lemma.

Lemma 6.1. For $q+1 \leq j_{1}, j_{2}<M_{1}(\alpha)$ and $\mathbf{a}=\left(a_{12}, \ldots, a_{1234}\right) \in \mathcal{A}$, we have

$$
\frac{n^{2\left(\tau_{j_{1}}(\boldsymbol{\alpha})+\tau_{j_{2}}(\boldsymbol{\alpha})\right)-\Psi(\mathbf{a}, \boldsymbol{\alpha})}}{n^{4 \tau_{k}(\boldsymbol{\alpha})-2 \tau_{q}(\boldsymbol{\alpha})}} \leq 1 \text {. }
$$

Proof. Notice that

$$
\begin{aligned}
D:= & 2\left(\tau_{j_{1}}(\boldsymbol{\alpha})+\tau_{j_{2}}(\boldsymbol{\alpha})\right)-\Psi(\mathbf{a}, \boldsymbol{\alpha}) \\
\leq & 2\left(\tau_{j_{1}}(\boldsymbol{\alpha})+\tau_{j_{2}}(\boldsymbol{\alpha})\right)-\tau_{a_{12}-1}(\boldsymbol{\alpha})-\tau_{a_{13}-1}(\boldsymbol{\alpha})-\tau_{a_{14}-1}(\boldsymbol{\alpha})-\tau_{a_{23}-1}(\boldsymbol{\alpha}) \\
& -\tau_{a_{24}-1}(\boldsymbol{\alpha})-\tau_{a_{34}-1}(\boldsymbol{\alpha})+\tau_{a_{123}-1}(\boldsymbol{\alpha})+\tau_{a_{124}-1}(\boldsymbol{\alpha})+\tau_{a_{134}-1}(\boldsymbol{\alpha})+\tau_{a_{234}-1}(\boldsymbol{\alpha}),
\end{aligned}
$$

and, by the choice of $j_{1}, j_{2}$, all the terms $\tau$. $(\boldsymbol{\alpha})$ in the right hand side are non-negative. Since the sequence $\left(\tau_{i}(\boldsymbol{\alpha}), i \geq-1\right)$ is unimodal-it increases until $i=k$ and then decreases-we have

$$
\begin{aligned}
& \tau_{a_{12}-1}(\boldsymbol{\alpha}) \geq \min \left(\tau_{j_{1}}(\boldsymbol{\alpha}), \tau_{a_{123}-1}(\boldsymbol{\alpha}) \vee \tau_{a_{124}-1}(\boldsymbol{\alpha})\right), \\
& \tau_{a_{13}-1}(\boldsymbol{\alpha}) \geq \min \left(\tau_{j_{1}}(\boldsymbol{\alpha}) \vee \tau_{j_{2}}(\boldsymbol{\alpha}), \tau_{a_{123}-1}(\boldsymbol{\alpha}) \vee \tau_{a_{134}-1}(\boldsymbol{\alpha})\right), \\
& \tau_{a_{14}-1}(\boldsymbol{\alpha}) \geq \min \left(\tau_{j_{1}}(\boldsymbol{\alpha}) \vee \tau_{j_{2}}(\boldsymbol{\alpha}), \tau_{a_{124}-1}(\boldsymbol{\alpha}) \vee \tau_{a_{134}-1}(\boldsymbol{\alpha})\right), \\
& \tau_{a_{23}-1}(\boldsymbol{\alpha}) \geq \min \left(\tau_{j_{1}}(\boldsymbol{\alpha}) \vee \tau_{j_{2}}(\boldsymbol{\alpha}), \tau_{a_{123}-1}(\boldsymbol{\alpha}) \vee \tau_{a_{234}-1}(\boldsymbol{\alpha})\right), \\
& \tau_{a_{24}-1}(\boldsymbol{\alpha}) \geq \min \left(\tau_{j_{1}}(\boldsymbol{\alpha}) \vee \tau_{j_{2}}(\boldsymbol{\alpha}), \tau_{a_{124}-1}(\boldsymbol{\alpha}) \vee \tau_{a_{234}-1}(\boldsymbol{\alpha})\right), \\
& \tau_{a_{34}-1}(\boldsymbol{\alpha}) \geq \min \left(\tau_{j_{2}}(\boldsymbol{\alpha}), \tau_{a_{134}-1}(\boldsymbol{\alpha}) \vee \tau_{a_{234}-1}(\boldsymbol{\alpha})\right) .
\end{aligned}
$$

Since $\mathbf{a} \in \mathcal{A}$, at least one of the 7 conditions in (6.16) holds. We will consider in detail what happens under condition $(i)$; the situation under the other conditions is similar. 
Under condition $(i)$ in (6.16) the first and the last bounds in (6.19) are supplemented by the bounds $\tau_{a_{12}-1}(\boldsymbol{\alpha}) \geq \tau_{q}(\boldsymbol{\alpha}), \tau_{a_{34}-1}(\boldsymbol{\alpha}) \geq \tau_{q}(\boldsymbol{\alpha})$. We now use the remaining 4 inequalities in (6.19). Note that $\tau_{a_{13}-1}(\boldsymbol{\alpha})$ "kills" (i.e., is at least as large as) $\tau_{j_{1}}(\boldsymbol{\alpha}), \tau_{j_{2}}(\boldsymbol{\alpha})$ or $\tau_{a_{123}-1}(\boldsymbol{\alpha})$. Similarly, $\tau_{a_{14}-1}(\boldsymbol{\alpha})$ "kills" $\tau_{j_{1}}(\boldsymbol{\alpha}), \tau_{j_{2}}(\boldsymbol{\alpha})$ or $\tau_{a_{134}-1}(\boldsymbol{\alpha})$. Further, $\tau_{a_{23}-1}(\boldsymbol{\alpha})$ "kills" $\tau_{j_{1}}(\boldsymbol{\alpha}), \tau_{j_{2}}(\boldsymbol{\alpha})$ or $\tau_{a_{234}-1}(\boldsymbol{\alpha})$. Finally, $\tau_{a_{24}-1}(\boldsymbol{\alpha})$ "kills" $\tau_{j_{1}}(\boldsymbol{\alpha}), \tau_{j_{2}}(\boldsymbol{\alpha})$ or $\tau_{a_{124}-1}(\boldsymbol{\alpha})$. This leaves 4 non-negative terms in the upper bound for $D$, neither of which exceeds $\tau_{k}(\boldsymbol{\alpha})$, so $D \leq 4 \tau_{k}(\boldsymbol{\alpha})-2 \tau_{q}(\boldsymbol{\alpha})$, as required.

Since $\mathcal{A}$ is parameterized by the 11 variables $a_{12}, \ldots, a_{1234}$, its cardinality does not exceed $\left(j_{1}+j_{2}+1\right)^{11}$. Hence, by Lemma 6.1 and (6.18)

$$
\frac{\mathbb{E}\left[\left(\chi_{n}^{(1)}(t)-\chi_{n}^{(1)}(s)\right)^{2}\left(\chi_{n}^{(1)}(s)-\chi_{n}^{(1)}(r)\right)^{2}\right]}{n^{4 \tau_{k}(\boldsymbol{\alpha})-2 \tau_{q}(\boldsymbol{\alpha})}} \leq \sum_{j_{1}=q+1}^{M_{1}(\boldsymbol{\alpha})-1} \sum_{j_{2}=q+1}^{M_{1}(\boldsymbol{\alpha})-1} \frac{F_{j_{1}, j_{2}}(t, s, r)}{n^{4 \tau_{k}(\boldsymbol{\alpha})-2 \tau_{q}(\boldsymbol{\alpha})}} \leq B(t-r)^{(1+\gamma)}
$$

for some $0<B<\infty$, as required for (6.13).

\section{Proofs of the limit theorems for the betti numbers in the critical dimension}

Once again, we start with the strong law of large numbers.

Proof of (4.2) in Theorem 4.1. For $0<T<\infty$, we have to demonstrate that

$$
\sup _{0 \leq t \leq T} \frac{\left|\beta_{k}(t)-\mathbb{E}\left(f_{k}\right)\right|}{\mathbb{E}\left(f_{k}\right)} \rightarrow 0 \text { a.s. }
$$

By the Morse inequalities

$$
f_{k}(t)-f_{k+1}(t)-f_{k-1}(t) \leq \beta_{k}(t) \leq f_{k}(t),
$$

we have

$$
\left|\beta_{k}(t)-\mathbb{E}\left(f_{k}\right)\right| \leq\left|f_{k}(t)-\mathbb{E}\left(f_{k}\right)\right|+f_{k+1}(t)+f_{k-1}(t) .
$$

By (6.1) with $j=k$, it is enough to prove that as $n \rightarrow \infty$,

$$
\sup _{0 \leq t \leq T} \frac{f_{k+1}(t)}{\mathbb{E}\left(f_{k}\right)} \rightarrow 0 \text { a.s. and } \sup _{0 \leq t \leq T} \frac{f_{k-1}(t)}{\mathbb{E}\left(f_{k}\right)} \rightarrow 0 \text { a.s. }
$$

This is, however, an immediate conclusion of (6.1) with $j=k \pm 1$, since by Proposition 3.1,

$$
\lim _{n \rightarrow \infty} \frac{\mathbb{E}\left(f_{k+1}\right)}{\mathbb{E}\left(f_{k}\right)}=\lim _{n \rightarrow \infty} \frac{\mathbb{E}\left(f_{k-1}\right)}{\mathbb{E}\left(f_{k}\right)}=0
$$

We continue with the functional central limit theorem for Betti numbers.

Proof of (4.4) in Theorem 4.2. For convenience, we drop the subscript $n$ in expressions such as $\beta_{j, n}$ for the duration of the proof. We start with introducing some terminology related to the connectivity of a simplicial complex. It is analogous to the terminology used in [12] and [7]. An $\ell$-dimensional simplicial complex $X$, is called pure if every face of $X$ is contained in an $\ell$-face. A simplicial complex $K$ is said to be strongly connected of order $\ell$ if the following two conditions hold:

- The $\ell$-skeleton of $K$ is pure. 
- Every pair of $\ell$-faces $\sigma, \tau \in K$, can be connected by a sequence of $\ell$-faces,

$$
\sigma=\sigma_{0}, \sigma_{1}, \ldots, \sigma_{j-1}, \sigma_{j}=\tau
$$

for some $j \geq 1$, such that $\operatorname{dim}\left(\sigma_{i} \cap \sigma_{i+1}\right)=\ell-1,0 \leq i \leq j-1$.

In this case, we will simply say that $K$ is an $\ell$-strongly connected simplicial complex. Note that the dimension of $K$ itself may be greater than $\ell$. We call an $\ell$-strongly connected subcomplex $K$ of $X$ maximal if there is no other $\ell$-strongly connected subcomplex $K^{\prime} \supset K$. We start with a useful estimate similar to the computation in [7], p.117.

Lemma 7.1. Let $K$ be a $(k+1)$-strongly connected simplicial complex on $j \geq k+3$ vertices with a non-zero $(k+1)$-st Betti number. Then, for $\sigma \subset[n]$ with $|\sigma|=j$,

$\mathbb{P}($ the restriction of $X([n], \mathbf{p})$ to $\sigma$ is isomorphic to $K)$

$$
\leq j ! \prod_{i=q}^{k+1} p_{i}^{\left(\begin{array}{c}
k+3 \\
i+1
\end{array}\right)}\left(\prod_{i=q}^{k+1} p_{i}^{\left(\begin{array}{c}
k+1 \\
i
\end{array}\right)}\right)^{j-k-3} \text {. }
$$

Proof. The argument consists of estimating the number of faces of different dimensions $K$ has to contain. We start by denoting by $m$ the number of the $(k+1)$-faces in $K$. We order these faces as follows. Fix an arbitrary $(k+1)$-cycle in $K$ and choose any $(k+1)$-face from this cycle to be $f_{1}$. Since $K$ is $(k+1)$-strongly connected, we can order the rest of the $(k+1)$-faces in the order $f_{1}, \ldots, f_{m}$ such that each $f_{p}, p>1$, has a $k$-dimensional intersection with at least one $f_{q}$ with $q<p$. This ordering of the $(k+1)$-faces induces an ordering on the vertices in $K$, as follows. First, let $v_{1}, \ldots, v_{k+2}$ be the vertices, chosen in an arbitrary order, in the support of $f_{1}$. Each vertex after $v_{k+2}$ corresponds to the addition of a $(k+1)$-face $f_{\ell}$; in that, it lies in the support of $f_{\ell}$ but is not contained in $f_{1} \cup \cdots \cup f_{\ell-1}$. Since each vertex of $K$ belongs to some $(k+1)$-face, we obtain, in this way, an ordering $v_{k+3}, \ldots, v_{j}$ of all remaining vertices in $K$. Note at this point that each vertex after $v_{k+2}$, for each $1 \leq i \leq k+1$, is a vertex of $\left(\begin{array}{c}k+1 \\ i\end{array}\right)$ of $i$-faces of some new $(k+1)$-face $f_{\ell}$ being considered at that point. We let

$$
c=\max \left\{k+3 \leq m \leq j: v_{m} \text { is a vertex of the initially fixed }(k+1) \text {-cycle }\right\}
$$

and note that $c$ is well defined since the cycle must contain at least $k+3$ vertices. The corresponding vertex $v_{c}$ is, actually, contained in at least $k+2$ faces of dimension $k+1$, just as other vertices in the initially fixed $(k+1)$-cycle. Furthermore, $v_{c}$ is contained in the fewest number of $i$-faces if it is a part of exactly $k+2$ faces of dimension $k+1$. The latter occurs when, excluding $v_{c}$, there are precisely $k+2$ other vertices in this cycle and they together form a $(k+1)$-face. Therefore, when $v_{c}$ entered our enumeration of the vertices, for each $1 \leq i \leq k+1$, it was a vertex of at least $\left(\begin{array}{c}k+2 \\ i\end{array}\right)$ new $i$-faces in $K$. We now see that for each $1 \leq i \leq k+1$,

- $f_{1}$ contains $\left(\begin{array}{c}k+2 \\ i+1\end{array}\right)$ distinct $i$-faces in $K$;

- each vertex in $\left\{v_{k+3}, \ldots, v_{j}\right\} \backslash\left\{v_{c}\right\}$ corresponds to $\left(\begin{array}{c}k+1 \\ i\end{array}\right)$ new distinct $i$-faces in $K$;

- $v_{c}$ corresponds to at least $\left(\begin{array}{c}k+2 \\ i\end{array}\right)$ new distinct $i$-faces in $K$.

Therefore, for each $1 \leq i \leq k+1, K$ contains at least

$$
\left(\begin{array}{c}
k+2 \\
i+1
\end{array}\right)+(j-k-3)\left(\begin{array}{c}
k+1 \\
i
\end{array}\right)+\left(\begin{array}{c}
k+2 \\
i
\end{array}\right)=\left(\begin{array}{c}
k+3 \\
i+1
\end{array}\right)+(j-k-3)\left(\begin{array}{c}
k+1 \\
i
\end{array}\right)
$$

$i$-faces. Finally, since there are $j$ ! ways of ordering vertices in $\sigma$, we get the assertion of the lemma. 
By (3.3), the already established convergence in (4.3) tells us that

$$
\left(\frac{\sum_{j=0}^{n-1}(-1)^{j} \beta_{j}(t)-\mathbb{E}\left(\sum_{j=0}^{n-1}(-1)^{j} \beta_{j}\right)}{\sqrt{\operatorname{Var}\left(f_{k}\right)}}, t \geq 0\right) \Rightarrow\left(Z_{k}(t), t \geq 0\right)
$$

in finite dimensional distributions. In order to prove convergence in finite dimensional distributions in (4.4), we need to show that all (normalized) Betti numbers except that of critical dimension are asymptotically negligible in (7.1). Proposition A.1 in the Appendix shows negligibility of the Betti numbers in dimension smaller than the critical dimension. Together with (7.1), this gives that

$$
\left(\frac{\sum_{j=k}^{n-1}(-1)^{j} \beta_{j}(t)-\mathbb{E}\left(\sum_{j=k}^{n-1}(-1)^{j} \beta_{j}\right)}{\sqrt{\operatorname{Var}\left(f_{k}\right)}}, t \geq 0\right) \Rightarrow\left(Z_{k}(t), t \geq 0\right) .
$$

Furthermore, by repeating the same argument as in (6.9), along with an obvious bound $\beta_{j} \leq f_{j}$, we obtain that

$$
\left(\frac{\sum_{j=M(\boldsymbol{\alpha})}^{n-1}(-1)^{j} \beta_{j}(t)-\mathbb{E}\left(\sum_{j=M(\boldsymbol{\alpha})}^{n-1}(-1)^{j} \beta_{j}\right)}{\sqrt{\operatorname{Var}\left(f_{k}\right)}}, t \geq 0\right) \rightarrow \mathbf{0},
$$

in finite-dimensional distributions, where $M(\boldsymbol{\alpha})$ is defined in (3.7) and $\mathbf{0}$ is the constant zero process. Hence, we can conclude that

$$
\left(\frac{\sum_{j=k}^{M(\boldsymbol{\alpha})-1}(-1)^{j} \beta_{j}(t)-\mathbb{E}\left(\sum_{j=k}^{M(\boldsymbol{\alpha})-1}(-1)^{j} \beta_{j}\right)}{\sqrt{\operatorname{Var}\left(f_{k}\right)}}, t \geq 0\right) \Rightarrow\left(Z_{k}(t), t \geq 0\right), \quad n \rightarrow \infty
$$

in finite-dimensional distributions.

Note that if $M(\boldsymbol{\alpha})=k+1$, then (4.4) is automatic, so only the case $M(\boldsymbol{\alpha})>k+1$ needs to be considered. It is, of course, sufficient to show that for any $j=k+1, \ldots, M(\alpha)-1, \operatorname{Var}\left(\beta_{j}\right)$ is negligible relative to $\operatorname{Var}\left(f_{k}\right)$ as $n \rightarrow \infty$. We will consider in detail the case $M(\boldsymbol{\alpha})=k+2$, and prove negligibility of the variance of $\beta_{k+1}$. If $M(\alpha)>k+2$, the higher-order Betti numbers can be treated in a similar way.

Our argument relies on an explicit representation of $\beta_{k+1}(t)$ given by

$$
\beta_{k+1}(t)=\beta_{k+1}(X([n], \mathbf{p} ; t))=\sum_{j=k+3}^{n} \sum_{r \geq 1} \sum_{\sigma \subset[n],|\sigma|=j} r \eta_{\sigma}^{(j, r, k+1)}(t),
$$

where $\eta_{\sigma}^{(j, r, k+1)}(t)$ is the indicator function of the event that $\sigma$ forms a maximal $(k+1)$-strongly connected subcomplex $X(\sigma, \mathbf{p} ; t)$, such that $\beta_{k+1}(X(\sigma, \mathbf{p} ; t))=r$. See Proposition A.6 for a formal derivation of (7.3). We often omit superscripts from the indicator if the context is clear enough. Note that the second sum over $r \geq 1$ is a sum of at most $\left(\begin{array}{c}j \\ k+2\end{array}\right)$ terms, because $\beta_{k+1}(X(\sigma, \mathbf{p} ; t))$ is bounded by the number of $(k+1)$-faces of $\sigma$, which itself is bounded by $\left(\begin{array}{c}j \\ k+2\end{array}\right)$.

As $M(\boldsymbol{\alpha})=k+2$, it follows that $\tau_{k+1}(\boldsymbol{\alpha})>0$, and we can find a positive integer $D$ such that

$$
D>\frac{k+2+\tau_{k+1}(\boldsymbol{\alpha})}{\psi_{k+1}(\boldsymbol{\alpha})-1}>0,
$$


and we use it to define a truncated version of the representation of the Betti number in (7.3) as

$$
\tilde{\beta}_{k+1}(t)=\tilde{\beta}_{k+1}(X([n], \mathbf{p} ; t))=\sum_{j=k+3}^{D+k+1} \sum_{r \geq 1} \sum_{\sigma \subset[n],|\sigma|=j} r \eta_{\sigma}^{(j, r, k+1)}(t) .
$$

As before, we write $\tilde{\beta}_{k+1}:=\tilde{\beta}_{k+1}(0)$ and $\eta_{\sigma}^{(j, r, k+1)}:=\eta_{\sigma}^{(j, r, k+1)}(0)$. We claim that

$$
\left(\frac{\beta_{k}(t)-\mathbb{E}\left(\beta_{k}\right)}{\sqrt{\operatorname{Var}\left(f_{k}\right)}}-\frac{\tilde{\beta}_{k+1}(t)-\mathbb{E}\left(\tilde{\beta}_{k+1}\right)}{\sqrt{\operatorname{Var}\left(f_{k}\right)}}, t \geq 0\right) \Rightarrow\left(Z_{k}(t), t \geq 0\right), \quad n \rightarrow \infty
$$

in finite-dimensional distributions. Indeed, by (7.2) with $M(\boldsymbol{\alpha})=k+2$, it is enough to prove that $\mathbb{E}\left(\beta_{k+1}-\tilde{\beta}_{k+1}\right) \rightarrow 0, n \rightarrow \infty$. Since the sum over $r \geq 1$ in (7.3) contains at most $\left(\begin{array}{c}j \\ k+2\end{array}\right)$ terms,

$$
\begin{aligned}
\mathbb{E}\left(\beta_{k+1}-\tilde{\beta}_{k+1}\right) & \leq \mathbb{E}\left[\sum_{j=D+k+2}^{n}\left(\begin{array}{c}
j \\
k+2
\end{array}\right) \sum_{\sigma \subset[n],|\sigma|=j} \sum_{r \geq 1} \eta_{\sigma}^{(j, r, k+1)}\right] \\
& \leq \mathbb{E}\left[n^{k+2} \sum_{j=D+k+2}^{n} \sum_{\sigma \subset[n],|\sigma|=j} \sum_{r \geq 1} \eta_{\sigma}^{(j, r, k+1)}\right] .
\end{aligned}
$$

Whenever a $(k+1)$-strongly connected subcomplex is formed on $j \geq D+k+2$ vertices, it contains a further $(k+1)$-strongly connected subcomplex on exactly $D+k+2$ vertices. Furthermore, no two different such maximal subcomplexes can contain the same $(k+1)$-strongly connected subcomplex on $D+k+2$ vertices. Therefore,

$$
\begin{aligned}
\mathbb{E}\left(\beta_{k+1}-\tilde{\beta}_{k+1}\right) & \leq n^{k+2}\left(\begin{array}{c}
n \\
D+k+2
\end{array}\right) \sum_{K:|K|=D+k+2} \mathbb{P}\left(\sigma_{D+k+2} \text { is isomorphic to } K\right) \\
& \leq \frac{n^{D+2 k+4}}{(D+k+2) !} \sum_{K:|K|=D+k+2} \mathbb{P}\left(\sigma_{D+k+2} \text { is isomorphic to } K\right),
\end{aligned}
$$

where $\sigma_{D+k+2}$ is the restriction of the complex to fixed $D+k+2$ vertices, and the sum above is taken over all isomorphism classes of $(k+1)$-strongly connected complexes on $D+k+2$ points. Note that the number of terms in this sum is independent of $n$. Any such complex $K$ contains at least $\left(\begin{array}{c}k+2 \\ i+1\end{array}\right)+D\left(\begin{array}{c}k+1 \\ i\end{array}\right)$ faces of dimension $i$ for each $1 \leq i \leq k+1$; this counting is presented in the proof of Lemma 8.1 in [7]. Hence,

$$
\mathbb{P}\left(\sigma_{D+k+2} \text { is isomorphic to } K\right) \leq(D+k+2) ! \prod_{i=q}^{k+1} p_{i}^{\left(\begin{array}{c}
k+2 \\
i+1
\end{array}\right)+D\left(\begin{array}{c}
k+1 \\
i
\end{array}\right)},
$$

and so, by (7.4),

$$
\mathbb{E}\left(\beta_{k+1}-\tilde{\beta}_{k+1}\right) \leq C n^{k+2+\tau_{k+1}(\boldsymbol{\alpha})-D\left(\psi_{k+1}(\boldsymbol{\alpha})-1\right)} \rightarrow 0, \quad n \rightarrow \infty .
$$

Thus, (7.5) follows and, by Chebyshev's inequality, the claim (4.4) is established once we check that

$$
\frac{\operatorname{Var}\left(\tilde{\beta}_{k+1}\right)}{\operatorname{Var}\left(f_{k}\right)} \rightarrow 0, \quad n \rightarrow \infty
$$


It suffices to show that for every $j=k+3, \ldots, D+k+1$ and $r \geq 1$, we have

$$
\frac{\operatorname{Var}\left(\sum_{\sigma \subset[n],|\sigma|=j} \eta_{\sigma}^{(j, r, k+1)}\right)}{\operatorname{Var}\left(f_{k}\right)} \rightarrow 0, \quad n \rightarrow \infty .
$$

Simplifying the notation, we get

$$
\begin{aligned}
& \operatorname{Var}\left(\sum_{\sigma \subset[n],|\sigma|=j} \eta_{\sigma}\right)=\sum_{\substack{\sigma \subset[n],|\sigma|=j}} \sum_{\substack{\tau \subset[n],|\tau|=j}}\left[\mathbb{E}\left(\eta_{\sigma} \eta_{\tau}\right)-\mathbb{E}\left(\eta_{\sigma}\right) \mathbb{E}\left(\eta_{\tau}\right)\right] \\
& =\sum_{\ell=0}^{j} \sum_{\substack{\sigma \subset[n],|\sigma|=j}} \sum_{\substack{\tau \subset[n],|\tau|=j}}\left[\mathbb{E}\left(\eta_{\sigma} \eta_{\tau}\right)-\mathbb{E}\left(\eta_{\sigma}\right) \mathbb{E}\left(\eta_{\tau}\right)\right] \mathbb{1}\{|\sigma \cap \tau|=\ell\} \\
& =\sum_{\ell=0}^{j}\left(\begin{array}{l}
n \\
j
\end{array}\right)\left(\begin{array}{l}
j \\
\ell
\end{array}\right)\left(\begin{array}{c}
n-j \\
j-\ell
\end{array}\right)\left[\mathbb{E}\left(\eta_{\sigma} \eta_{\tau}\right)-\mathbb{E}\left(\eta_{\sigma}\right) \mathbb{E}\left(\eta_{\tau}\right)\right] \mathbb{1}\{|\sigma \cap \tau|=\ell\} .
\end{aligned}
$$

We consider six cases, depending on the value of $\ell:=|\sigma \cap \tau|$.

(I) $\ell \in\{0, \ldots, q-2\}$.

We claim that in this case the events underlying the indicator functions $\eta_{\sigma}$ and $\eta_{\tau}$ are independent, so that the corresponding terms have no contribution to the numerator in (7.6). Indeed, the event underlying $\eta_{\sigma}$ can be stated as saying that the restriction of the complex to $\sigma$ is a $(k+1)$-strongly connected subcomplex with Betti number in dimension $k+1$ equal to $r$ and that no $(k+1)$-simplex carried by $\sigma$ has $k+1$ common vertices, i.e., a common $k$-face, with a $(k+1)$-simplex not carried by $\sigma$. We also have an analogous description of the event underlying $\eta_{\tau}$. Stated this way, it is clear if a face $s_{1}$ plays a role in the former event, and a face $s_{2}$ plays a role in the latter event, then these faces have at most $q$ vertices in common and, hence, the restrictions of the complex to these faces are independent.

(II) $\ell=q-1$.

First, let

$$
\gamma_{k}:=\prod_{i=q}^{k+1} p_{i}^{\left(\begin{array}{c}
k+1 \\
i
\end{array}\right)}=n^{-\psi_{k+1}(\boldsymbol{\alpha})}
$$

denote the probability that a fixed $k$-face and a vertex not in that face form a $(k+1)$-simplex. For $j \in\{k+3, \ldots, D+k+1\}$ and $r \geq 1$, let $K$ denote a fixed $(k+1)$-strongly connected complex on $j$ vertices whose Betti number in dimension $k+1$ is equal to $r$. For $\sigma \subset[n]$ with $|\sigma|=j$, let $A_{K}$ be the event that the restriction of the complex to $\sigma$ is isomorphic to $K$, and define $q_{K}:=\mathbb{P}\left(A_{K}\right)$.

We first claim that, for every $\sigma \subset[n]$ with $|\sigma|=j$,

$$
\mathbb{E}\left(\eta_{\sigma}\right)=\sum_{K:|K|=j} q_{K}\left(1-s_{K} \gamma_{k}+u_{K}\right)^{n-j},
$$

where the sum is taken over all $(k+1)$-strongly connected complexes, up to an isomorphism class, such that the Betti number in dimension $k+1$ is equal to $r$. Moreover, $s_{K}$ is the number of $k$-faces in $K$, and $u_{K}=\mathcal{O}\left(\gamma_{k}\right)$ as functions of $n$, i.e., there exists $C>0$ such that $u_{K} / \gamma_{k}<C$ for all $n \geq 1$ and all $K$. Note that $q_{K}, \gamma_{k}$, and $u_{K}$ depend on $n$, whereas $s_{K}$ is independent of $n$. For the proof of (7.7), write

$$
\mathbb{E}\left(\eta_{\sigma}\right)=\sum_{K:|K|=j} q_{K} \mathbb{P}\left(\sigma \text { is maximal } \mid A_{K}\right) .
$$


Let us fix a vertex $v \in \sigma^{c}$. By the inclusion-exclusion formula, the probability of forming at least one $(k+1)$-simplex between $v$ and a $k$-face in $\sigma$, can be written as $s_{K} \gamma_{k}-u_{K}$. The largest term in $u_{K}$ corresponds to $v$ forming two $(k+1)$-simplices with $k$-faces $f_{1}$ and $f_{2}$ respectively, such that $\operatorname{dim}\left(f_{1} \cap f_{2}\right)=k-1$. Therefore, the largest term in $u_{K}$ is of the order $\gamma_{k}^{2} \gamma_{k-1}^{-1}=\mathcal{O}\left(\gamma_{k}\right)$. Since there are $n-j$ vertices in $\sigma^{c}$, we have

$\mathbb{P}\left(\sigma\right.$ is maximal $\left.\mid A_{K}\right)=\left(1-s_{K} \gamma_{k}+u_{K}\right)^{n-j}$,

and (7.7) follows as required.

Next, let $K, K^{\prime}$ be fixed $(k+1)$-strongly connected complexes on $j$ vertices with Betti number in dimension $k+1$ equal to $r$. Denote by $A_{K, K^{\prime}}$ the event that the restriction of the complex to $\sigma$ and that to $\tau$ are isomorphic to $K$ and $K^{\prime}$, respectively. It then follows from (7.7) that

$$
\begin{aligned}
& {\left[\mathbb{E}\left(\eta_{\sigma} \eta_{\tau}\right)-\mathbb{E}\left(\eta_{\sigma}\right) \mathbb{E}\left(\eta_{\tau}\right)\right] \mathbb{1}\{|\sigma \cap \tau|=q-1\}} \\
& =\sum_{K:|K|=j} \sum_{K^{\prime}:\left|K^{\prime}\right|=j}\left[\mathbb{P}\left(\sigma \text { and } \tau \text { are maximal } \mid A_{K, K^{\prime}}\right) \mathbb{P}\left(A_{K, K^{\prime}}\right)\right. \\
& \left.\quad-q_{K} q_{K^{\prime}}\left(1-s_{K} \gamma_{k}+u_{K}\right)^{n-j}\left(1-s_{K^{\prime}} \gamma_{k}+u_{K^{\prime}}\right)^{n-j}\right] \mathbb{1}\{|\sigma \cap \tau|=q-1\},
\end{aligned}
$$

where the sums are again taken over all $(k+1)$-strongly connected complexes whose Betti numbers in dimension $k+1$ are equal to $r$, and $s_{K^{\prime}}, u_{K^{\prime}}$ are defined analogously to those for $K$. Since $|\sigma \cap \tau|=q-1$ and all the $(q-2)$-faces exist with probability one, we have $\mathbb{P}\left(A_{K, K^{\prime}}\right)=q_{K} q_{K^{\prime}}$. For every $v \in(\sigma \cup \tau)^{c}$, let $B_{v}$ be the event that $v$ forms a $(k+1)$-simplex with a $k$-face in $\sigma \cup \tau$. Further, let $D_{1}$ denote the event that at least one $(k+1)$-simplex exists between a $k$-face in $\sigma$ and a point in $\tau \backslash(\sigma \cap \tau)$, and $D_{2}$ is an event obtained by switching the role of $\sigma$ and $\tau$. Then, by independence we see that

$$
\begin{aligned}
\mathbb{P}\left(\sigma \text { and } \tau \text { are maximal } \mid A_{K, K^{\prime}}\right) & =\mathbb{P}\left(\left(\bigcap_{v \in(\sigma \cup \tau)^{c}} B_{v}^{c}\right) \cap D_{1}^{c} \cap D_{2}^{c} \mid A_{K, K^{\prime}}\right) \\
& =\prod_{v \in(\sigma \cup \tau)^{c}}\left(1-\mathbb{P}\left(B_{v} \mid A_{K, K^{\prime}}\right)\right) \mathbb{P}\left(D_{1}^{c} \cap D_{2}^{c} \mid A_{K, K^{\prime}}\right) .
\end{aligned}
$$

By the inclusion-exclusion formula, we have

$$
\mathbb{P}\left(B_{v} \mid A_{K, K^{\prime}}\right)=\left(s_{K}+s_{K^{\prime}}\right) \gamma_{k}-u_{K}-u_{K^{\prime}}-s_{K} s_{K^{\prime}} \gamma_{k}^{2}+s_{K} \gamma_{k} u_{K^{\prime}}+s_{K^{\prime}} \gamma_{k} u_{K}-u_{K} u_{K^{\prime}}=: a_{K, K^{\prime}} .
$$

Indeed, the probabilities that $v$ forms $(k+1)$-simplices with multiple $k$-faces in $\sigma$ are grouped into $u_{K}$, while the probabilities that $v$ forms $(k+1)$-simplices with multiple $k$-faces in $\tau$ are grouped into $u_{K^{\prime}}$. Moreover, the probabilities that $v$ forms $(k+1)$-simplices with both $k$-faces in $\sigma$ and those in $\tau$, are grouped into one of the last four terms in (7.10). Above, we have also exploited the fact that the events concerning $v$ forming $(k+1)$-simplices with $k$-faces in $\sigma$ are independent from events concerning $v$ forming $(k+1)$-simplices with $k$-faces in $\tau$.

Noting that there are $n-2 j+q-1$ points in $(\sigma \cup \tau)^{c}$, the right hand side of (7.8) is equal to

$$
\sum_{K:|K|=j} \sum_{K^{\prime}:\left|K^{\prime}\right|=j} q_{K} q_{K^{\prime}}\left(1-a_{K, K^{\prime}}\right)^{n-2 j+q-1}\left[\mathbb{P}\left(D_{1}^{c} \cap D_{2}^{c} \mid A_{K, K^{\prime}}\right)-\left(1-a_{K, K^{\prime}}\right)^{j-q+1}\right]
$$


By the binomial expansion, it is easy to see that

$$
\begin{aligned}
& \left(1-a_{K, K^{\prime}}\right)^{n-2 j+q-1}=\left(1-\mathcal{O}\left(\gamma_{k}\right)\right)^{n-2 j+q-1}=\mathcal{O}(1), \\
& \left(1-a_{K, K^{\prime}}\right)^{j-q+1}=1-(j-q+1) a_{K, K^{\prime}}+\mathcal{O}\left(\gamma_{k}^{2}\right),
\end{aligned}
$$

and, further,

$$
\begin{aligned}
& \mathbb{P}\left(D_{1} \mid A_{K, K^{\prime}}\right)=1-\left(1-s_{K} \gamma_{k}+u_{K}\right)^{j-q+1}=(j-q+1)\left(s_{K} \gamma_{k}-u_{K}\right)-\mathcal{O}\left(\gamma_{k}^{2}\right), \\
& \mathbb{P}\left(D_{2} \mid A_{K, K^{\prime}}\right)=1-\left(1-s_{K^{\prime}} \gamma_{k}+u_{K^{\prime}}\right)^{j-q+1}=(j-q+1)\left(s_{K^{\prime}} \gamma_{k}-u_{K^{\prime}}\right)-\mathcal{O}\left(\gamma_{k}^{2}\right) .
\end{aligned}
$$

Suppose now that

there exist two $k$-faces $f_{1} \subset \sigma$ and $f_{2} \subset \tau$ such that $\left|f_{1} \cap f_{2}\right|=q-1$.

Under (7.12), we claim that

$$
\mathbb{P}\left(D_{1} \cap D_{2} \mid A_{K, K^{\prime}}\right)=\mathcal{O}\left(\gamma_{k}^{2} p_{q}^{-1}\right) .
$$

Indeed, the largest term in the right hand side corresponds to the case in which a vertex in $f_{1} \backslash\left(f_{1} \cap f_{2}\right)$ forms a $(k+1)$-simplex with $f_{2}$, and a vertex in $f_{2} \backslash\left(f_{1} \cap f_{2}\right)$ forms a $(k+1)$ simplex with $f_{1}$. Because of a double-count of a $q$-face consisting of the vertices in $f_{1} \cap f_{2}$ and the two selected vertices, the largest rate is of order $\gamma_{k}^{2} p_{q}^{-1}$. By combining all these results, it is now straightforward to get that

$$
\mathbb{P}\left(D_{1}^{c} \cap D_{2}^{c} \mid A_{K, K^{\prime}}\right)-\left(1-a_{K, K^{\prime}}\right)^{j-q+1}=\mathcal{O}\left(\gamma_{k}^{2} p_{q}^{-1}\right) .
$$

If (7.12) does not hold, the same analysis gives the behavior as in (7.13), but with a smaller correction term; $\mathcal{O}\left(\gamma_{k}^{2}\right)$ instead of $\mathcal{O}\left(\gamma_{k}^{2} p_{q}^{-1}\right)$. From all of these results, (7.11) can be written as

$$
C \sum_{\substack{K:|K|=j \\(7.12) \text { holds }}} \sum_{K^{\prime}:\left|K^{\prime}\right|=j} q_{K} q_{K^{\prime}} \mathcal{O}\left(\gamma_{k}^{2} p_{q}^{-1}\right),
$$

and, thus,

$$
\begin{aligned}
& \left(\begin{array}{l}
n \\
j
\end{array}\right)\left(\begin{array}{c}
j \\
q-1
\end{array}\right)\left(\begin{array}{c}
n-j \\
j-q+1
\end{array}\right)\left[\mathbb{E}\left(\eta_{\sigma} \eta_{\tau}\right)-\mathbb{E}\left(\eta_{\sigma}\right) \mathbb{E}\left(\eta_{\tau}\right)\right] \mathbb{1}\{|\sigma \cap \tau|=q-1\} \\
& \leq C \sum_{\substack{K:|K|=j \\
(7.12) \text { holds }}} \sum_{K^{\prime}:\left|K^{\prime}\right|=j} n^{2 j-q+1} q_{K} q_{K^{\prime}} \mathcal{O}\left(\gamma_{k}^{2} p_{q}^{-1}\right) .
\end{aligned}
$$

By Lemma 7.1,

$$
n^{j} q_{K} \leq C n^{j} \prod_{i=q}^{k+1} p_{i}^{\left(\begin{array}{c}
k+3 \\
i+1
\end{array}\right)}\left(\prod_{i=q}^{k+1} p_{i}^{\left({ }^{k+1}\right)}\right)^{j-k-3}=C n^{\tau_{k+2}(\boldsymbol{\alpha})+\alpha_{k+2}}\left(n^{1-\psi_{k+1}(\boldsymbol{\alpha})}\right)^{j-k-3} .
$$

Since $\psi_{k+1}(\boldsymbol{\alpha})>1$ and $j \geq k+3$, we get $\left(n^{1-\psi_{k+1}(\boldsymbol{\alpha})}\right)^{j-k-3} \leq 1$, and hence,

$$
n^{2 j} q_{K} q_{K^{\prime}} \leq C n^{2\left(\tau_{k+2}(\boldsymbol{\alpha})+\alpha_{k+2}\right)} \text {. }
$$

It now remains to check that

$$
\frac{n^{2\left(\tau_{k+2}(\boldsymbol{\alpha})+\alpha_{k+2}\right)} \mathcal{O}\left(n^{-q+1} \gamma_{k}^{2} p_{q}^{-1}\right)}{\operatorname{Var}\left(f_{k}\right)} \rightarrow 0 \text { as } n \rightarrow \infty .
$$


But this actually follows, since by Proposition 3.1 and (2.12), the expression on the left hand side is bounded by

$$
C n^{2\left(\tau_{k+1}(\boldsymbol{\alpha})-\tau_{k}(\boldsymbol{\alpha})\right)} \mathcal{O}\left(n^{\tau_{q}(\boldsymbol{\alpha})-q+1} \gamma_{k}^{2} p_{q}^{-1}\right)=\operatorname{Co}(1) \mathcal{O}\left(n^{2\left(1-\psi_{k+1}(\boldsymbol{\alpha})\right)}\right) \rightarrow 0, \quad n \rightarrow \infty .
$$

(III) $\ell=q$.

The case $\ell=q$ is similar but easier. Using the same notation as in Case $(I I)$, we once again consider

$$
\begin{aligned}
& {\left[\mathbb{E}\left(\eta_{\sigma} \eta_{\tau}\right)-\mathbb{E}\left(\eta_{\sigma}\right) \mathbb{E}\left(\eta_{\tau}\right)\right] \mathbb{1}\{|\sigma \cap \tau|=q\}} \\
& =\sum_{K:|K|=j} \sum_{K^{\prime}:\left|K^{\prime}\right|=j}\left[\mathbb{P}\left(\sigma \text { and } \tau \text { are maximal } \mid A_{K, K^{\prime}}\right) \mathbb{P}\left(A_{K, K^{\prime}}\right)\right. \\
& \left.\quad-q_{K} q_{K^{\prime}}\left(1-s_{K} \gamma_{k}+u_{K}\right)^{n-j}\left(1-s_{K^{\prime}} \gamma_{k}+u_{K^{\prime}}\right)^{n-j}\right] \mathbb{1}\{|\sigma \cap \tau|=q\} .
\end{aligned}
$$

Since $|\sigma \cap \tau|=q$ and all the $(q-1)$-faces exist with probability one, we still get $\mathbb{P}\left(A_{K, K^{\prime}}\right)=$ $q_{K} q_{K^{\prime}}$. By the same reasoning as before, we only consider the situation that

$$
\text { there exist two } k \text {-faces } f_{1} \subset \sigma \text { and } f_{2} \subset \tau \text { such that }\left|f_{1} \cap f_{2}\right|=q \text {. }
$$

Under this assumption, for each $v \in(\sigma \cup \tau)^{c}$, the inclusion-exclusion formula gives that

$$
\mathbb{P}\left(B_{v} \mid A_{K, K^{\prime}}\right)=\left(s_{K}+s_{K^{\prime}}\right) \gamma_{k}-u_{K}-u_{K^{\prime}}-\mathcal{O}\left(\gamma_{k}^{2} p_{q}^{-1}\right),
$$

The largest term in the big- $\mathcal{O}$ expression is associated with the case in which $v$ forms two $(k+1)$-simplices with $f_{1}$ and $f_{2}$, respectively. By (7.9), we see that

$$
\begin{aligned}
& \mathbb{P}\left(\sigma \text { and } \tau \text { are maximal } \mid A_{K, K^{\prime}}\right) \leq \prod_{v \in(\sigma \cup \tau)^{c}}\left(1-\mathbb{P}\left(B_{v} \mid A_{K, K^{\prime}}\right)\right) \\
& \quad=\left(1-\left(s_{K}+s_{K^{\prime}}\right) \gamma_{k}+u_{K}+u_{K^{\prime}}+\mathcal{O}\left(\gamma_{k}^{2} p_{q}^{-1}\right)\right)^{n-2 j+q} \\
& \quad=\left(1-\left(s_{K}+s_{K^{\prime}}\right) \gamma_{k}+u_{K}+u_{K^{\prime}}\right)^{n}\left(1+\mathcal{O}\left(\gamma_{k}\right)\right)^{-(2 j-q)}\left(1+\mathcal{O}\left(\gamma_{k}^{2} p_{q}^{-1}\right)\right)^{n} \\
& \quad=\left(1-\left(s_{K}+s_{K^{\prime}}\right) \gamma_{k}+u_{K}+u_{K^{\prime}}\right)^{n}\left(1+\mathcal{O}\left(\gamma_{k} p_{q}^{-1}\right)\right) .
\end{aligned}
$$

Here, we have made use of the following facts: $\gamma_{k}^{2} p_{q}^{-1}=\mathcal{O}\left(\gamma_{k}\right),\left(1+O\left(\gamma_{k}\right)\right)^{-(2 j-q)}=1+O\left(\gamma_{k}\right)$, and $\left(1+\mathcal{O}\left(\gamma_{k}^{2} p_{q}^{-1}\right)\right)^{n}=1+\mathcal{O}\left(n \gamma_{k}^{2} p_{q}^{-1}\right)=1+\mathcal{O}\left(\gamma_{k} p_{q}^{-1}\right)$.

Similarly, we derive that

$$
\begin{aligned}
\left(1-s_{K} \gamma_{k}+u_{K}\right)^{n-j}\left(1-s_{K^{\prime}} \gamma_{k}+u_{K^{\prime}}\right)^{n-j}= & \left(1-\left(s_{K}+s_{K^{\prime}}\right) \gamma_{k}+u_{K}+u_{K^{\prime}}+\mathcal{O}\left(\gamma_{k}^{2}\right)\right)^{n-j} \\
= & \left(1-\left(s_{K}+s_{K^{\prime}}\right) \gamma_{k}+u_{K}+u_{K^{\prime}}\right)^{n} \\
& \times\left(1+\mathcal{O}\left(\gamma_{k}\right)\right) .
\end{aligned}
$$

Putting all these results together, along with the binomial expansion $\left(1-\left(s_{K}+s_{K^{\prime}}\right) \gamma_{k}+u_{K}+\right.$ $\left.u_{K^{\prime}}\right)^{n}=\mathcal{O}(1)$ as $n \rightarrow \infty$, we can conclude that

$$
\begin{aligned}
& \left(\begin{array}{l}
n \\
j
\end{array}\right)\left(\begin{array}{l}
j \\
q
\end{array}\right)\left(\begin{array}{c}
n-j \\
j-q
\end{array}\right)\left[\mathbb{E}\left(\eta_{\sigma} \eta_{\tau}\right)-\mathbb{E}\left(\eta_{\sigma}\right) \mathbb{E}\left(\eta_{\tau}\right)\right] \mathbb{1}\{|\sigma \cap \tau|=q\} \\
& \leq C \sum_{\substack{K:|K|=j \\
(7.15) \text { holds }}} \sum_{K^{\prime}:\left|K^{\prime}\right|=j} n^{2 j-q} q_{K} q_{K^{\prime}} \mathcal{O}\left(\gamma_{k} p_{q}^{-1}\right) .
\end{aligned}
$$


Using Lemma 7.1 as in (7.14), it follows that the right hand side above can be bounded by $C n^{2\left(\tau_{k+2}(\boldsymbol{\alpha})+\alpha_{k+2}\right)} \mathcal{O}\left(n^{-q} \gamma_{k} p_{q}^{-1}\right)$. Finally, Proposition 3.1 and (2.12) help to conclude that

$$
\begin{aligned}
\frac{n^{2\left(\tau_{k+2}(\boldsymbol{\alpha})+\alpha_{k+2}\right)} \mathcal{O}\left(n^{-q} \gamma_{k} p_{q}^{-1}\right)}{\operatorname{Var}\left(f_{k}\right)} & \leq C n^{2\left(\tau_{k+1}(\boldsymbol{\alpha})-\tau_{k}(\boldsymbol{\alpha})\right)} \mathcal{O}\left(n^{\tau_{q}(\boldsymbol{\alpha})-q-\psi_{k+1}(\boldsymbol{\alpha})+\alpha_{q}}\right) \\
& =\operatorname{Co}(1) \mathcal{O}\left(n^{1-\psi_{k+1}(\boldsymbol{\alpha})}\right) \rightarrow 0, \quad n \rightarrow \infty
\end{aligned}
$$

$(I V) \ell \in\{q+1, \ldots, k+2\}$.

Note first that

$$
\begin{aligned}
\left(\begin{array}{l}
n \\
j
\end{array}\right)\left(\begin{array}{l}
j \\
\ell
\end{array}\right)\left(\begin{array}{c}
n-j \\
j-\ell
\end{array}\right)\left[\mathbb{E}\left(\eta_{\sigma} \eta_{\tau}\right)-\mathbb{E}\left(\eta_{\sigma}\right) \mathbb{E}\left(\eta_{\tau}\right)\right] \mathbb{1}\{|\sigma \cap \tau|=\ell\} \\
\leq n^{2 j-\ell} \mathbb{E}\left(\eta_{\sigma} \eta_{\tau}\right) \mathbb{1}\{|\sigma \cap \tau|=\ell\} \\
\leq n^{2 j-\ell} \sum_{K:|K|=j} \sum_{K^{\prime}:\left|K^{\prime}\right|=j} \mathbb{P}\left(A_{K, K^{\prime}}\right) \mathbb{1}\{|\sigma \cap \tau|=\ell\} .
\end{aligned}
$$

where $A_{K, K^{\prime}}$ is as in Case $(I I)$. Since there are finitely many isomorphism classes of $(k+1)$ strongly connected complexes on $j$ vertices, we only have to show that for all such $K, K^{\prime}$ with $|\sigma \cap \tau|=\ell$,

$$
\left(\operatorname{Var}\left(f_{k}\right)\right)^{-1} n^{2 j-\ell} \mathbb{P}\left(A_{K, K^{\prime}}\right) \rightarrow 0, \quad n \rightarrow \infty .
$$

By Lemma 7.1,

$$
\mathbb{P}\left(A_{K, K^{\prime}}\right) \leq C\left[\prod_{i=q}^{k+1} p_{i}^{\left(\begin{array}{l}
k+3 \\
i+1
\end{array}\right)}\left(\prod_{i=q}^{k+1} p_{i}^{\left(\begin{array}{c}
k+1 \\
i
\end{array}\right)}\right)^{j-k-3}\right]^{2} \times \prod_{i=q}^{k+1} p_{i}^{-\left(\begin{array}{c}
\ell \\
i+1
\end{array}\right)},
$$

with the last factor accounting for the faces on the vertices common to $\sigma$ and $\tau$. We conclude that

$$
\begin{aligned}
& n^{2 j-\ell} \mathbb{P}\left(A_{K, K^{\prime}}\right) \leq C n^{2 j-\ell} \prod_{i=q}^{k+1} p_{i}^{2\left(\begin{array}{c}
k+3 \\
i+1
\end{array}\right)-\left(\begin{array}{c}
\ell \\
i+1
\end{array}\right)}\left(\prod_{i=q}^{k+1} p_{i}^{\left(\begin{array}{c}
k+1 \\
i
\end{array}\right)}\right)^{2(j-k-3)} \\
= & C n^{2\left(\tau_{k+2}(\boldsymbol{\alpha})+\alpha_{k+2}\right)-\tau_{\ell-1}(\boldsymbol{\alpha})}\left(n^{1-\psi_{k+1}(\boldsymbol{\alpha})}\right)^{2(j-k-3)} \leq C n^{2\left(\tau_{k+2}(\boldsymbol{\alpha})+\alpha_{k+2}\right)-\tau_{\ell-1}(\boldsymbol{\alpha})} .
\end{aligned}
$$

By Proposition 3.1 and (2.12),

$$
\frac{n^{2\left(\tau_{k+2}(\boldsymbol{\alpha})+\alpha_{k+2}\right)-\tau_{\ell-1}(\boldsymbol{\alpha})}}{\operatorname{Var}\left(f_{k}\right)} \leq C n^{2\left(\tau_{k+1}(\boldsymbol{\alpha})-\tau_{k}(\boldsymbol{\alpha})\right)+\left(\tau_{q}(\boldsymbol{\alpha})-\tau_{\ell-1}(\boldsymbol{\alpha})\right)} \rightarrow 0
$$

because the exponent is clearly negative if $\ell \in\{q+1, \ldots, k+1\}$, and it is still true in the case $\ell=k+2$, because

$$
2\left(\tau_{k+1}(\boldsymbol{\alpha})-\tau_{k}(\boldsymbol{\alpha})\right)+\left(\tau_{q}(\boldsymbol{\alpha})-\tau_{\ell-1}(\boldsymbol{\alpha})\right)=\left(\tau_{k+1}(\boldsymbol{\alpha})-\tau_{k}(\boldsymbol{\alpha})\right)+\left(\tau_{q}(\boldsymbol{\alpha})-\tau_{k}(\boldsymbol{\alpha})\right)<0 .
$$

(V) $\ell \in\{k+3, \ldots, j-1\}$.

It is still sufficient to prove (7.16), which we presently do. We note that

$$
\mathbb{P}\left(A_{K, K^{\prime}}\right)=\mathbb{P}(\text { the complex restricted to } \sigma \text { is isomorphic to } K)
$$

$\mathbb{P}\left(\right.$ the complex restricted to $\tau$ is isomorphic to $\left.K^{\prime}\right) D\left(K, K^{\prime}\right)$,

where $D\left(K, K^{\prime}\right)$ is the correction term, resulting from the fact that some of the faces in the restriction of the complex to $\sigma \cap \tau$ are used in both $K$ and $K^{\prime}$. Hence, for each fixed $\ell$, we obtain 
an upper bound on $\mathbb{P}\left(A_{K, K^{\prime}}\right)$ by considering the worst case scenario (from the perspective of showing (7.16)).

To see how it works, consider the case $\ell=k+3$. Clearly, the worst case scenario is when both $K$ and $K^{\prime}$ have the least number of $i$-faces for $q \leq i \leq k+1$; further, in the complex restricted to $\sigma \cap \tau$, there is a maximum overlapping of faces. However, since $K$ and $K^{\prime}$ are $(k+1)$-strongly connected, even in this worst case scenario, the complex restricted to the $k+3$ vertices in $\sigma \cap \tau$ should have at least two $(k+1)$-faces; of course, these two may have a common shared $k$-face.

Hence,

$$
D\left(K, K^{\prime}\right)=\prod_{i=q}^{k+1} p_{i}^{-\left(\begin{array}{c}
k+2 \\
i+1
\end{array}\right)} \prod_{i=q}^{k+1} p_{i}^{-\left(\begin{array}{c}
k+1 \\
i
\end{array}\right)}
$$

so, by Lemma 7.1, we have

$$
n^{2 j-(k+3)} \mathbb{P}\left(A_{K, K^{\prime}}\right) \leq C n^{2 j-(k+3)}\left[\prod_{i=q}^{k+1} p_{i}^{\left(\begin{array}{c}
k+3 \\
i+1
\end{array}\right)}\left(\prod_{i=q}^{k+1} p_{i}^{\left(\begin{array}{c}
k+1 \\
i
\end{array}\right)}\right)^{j-k-3}\right]^{2} \prod_{i=q}^{k+1} p_{i}^{-\left(\begin{array}{c}
k+2 \\
i+1
\end{array}\right)} \prod_{i=q}^{k+1} p_{i}^{-\left(\begin{array}{c}
k+1 \\
i
\end{array}\right)} .
$$

Suppose next that $\ell=k+4$. In the worst case scenario now, the restriction of the complex to $k+3$ (out of the $k+4$ ) common points of the intersection should have the same setup as in the previous case, while the last $(k+4)$ th common point should form a $(k+1)$-simplex with one of the two $(k+1)$-simplices constructed before. Once again, this is the minimal requirement since both $K$ and $K^{\prime}$ are $(k+1)$-strongly connected. Hence,

$$
D_{\sigma, \tau}\left(K, K^{\prime}\right)=\prod_{i=q}^{k+1} p_{i}^{-\left(\begin{array}{c}
k+2 \\
i+1
\end{array}\right)}\left(\prod_{i=q}^{k+1} p_{i}^{-\left(\begin{array}{c}
k+1 \\
i
\end{array}\right)}\right)^{2}
$$

so, by Lemma 7.1

$$
\begin{aligned}
& n^{2 j-(k+4)} \mathbb{P}\left(A_{K, K^{\prime}}\right) \\
& \quad \leq C n^{2 j-(k+4)}\left[\prod_{i=q}^{k+1} p_{i}^{\left(\begin{array}{c}
k+3 \\
i+1
\end{array}\right)}\left(\prod_{i=q}^{k+1} p_{i}^{\left(\begin{array}{c}
k+1 \\
i
\end{array}\right)}\right)^{j-k-3}\right]^{2} \prod_{i=q}^{k+1} p_{i}^{-\left(\begin{array}{c}
k+2 \\
i+1
\end{array}\right)}\left(\prod_{i=q}^{k+1} p_{i}^{-\left(\begin{array}{c}
k+1 \\
i
\end{array}\right)}\right)^{2} .
\end{aligned}
$$

Proceeding in the same manner for any $\ell \in\{k+3, \ldots, j-1\}$, we see that

$$
n^{2 j-\ell} \mathbb{P}\left(A_{K, K^{\prime}}\right) \leq C n^{2 j-\ell}\left[\prod_{i=q}^{k+1} p_{i}^{\left(\begin{array}{c}
k+3 \\
i+1
\end{array}\right)}\left(\prod_{i=q}^{k+1} p_{i}^{\left(\begin{array}{c}
k+1 \\
i
\end{array}\right)}\right)^{j-k-3}\right]^{2} \prod_{i=q}^{k+1} p_{i}^{-\left(\begin{array}{c}
k+2 \\
i+1
\end{array}\right)}\left(\prod_{i=q}^{k+1} p_{i}^{-\left(\begin{array}{c}
k+1 \\
i
\end{array}\right)}\right)^{\ell-(k+2)} .
$$

Therefore, as before,

$$
\begin{aligned}
& n^{2 j-\ell}\left[\prod_{i=q}^{k+1} p_{i}^{\left(\begin{array}{c}
k+3 \\
i+1
\end{array}\right)}\left(\prod_{i=q}^{k+1} p_{i}^{\left(\begin{array}{c}
k+1 \\
i
\end{array}\right)}\right)^{j-k-3}\right]^{2} \prod_{i=q}^{k+1} p_{i}^{-\left(\begin{array}{c}
k+2 \\
i+1
\end{array}\right)}\left(\prod_{i=q}^{k+1} p_{i}^{-\left(\begin{array}{c}
k+1 \\
i
\end{array}\right)}\right)^{\ell-(k+2)} \\
& =n^{2\left(\tau_{k+2}(\boldsymbol{\alpha})+\alpha_{k+2}\right)-\tau_{k+1}(\boldsymbol{\alpha})}\left(n^{1-\psi_{k+1}(\boldsymbol{\alpha})}\right)^{2 j-k-\ell-4} \\
& \leq n^{2\left(\tau_{k+2}(\boldsymbol{\alpha})+\alpha_{k+2}\right)-\tau_{k+1}(\boldsymbol{\alpha})}
\end{aligned}
$$

which is the same bound as that for $\ell=k+2$ in the previous case. Thus, we get (7.16), as desired.

$(V I) \ell=j$. 
We again prove (7.16), this time only with $K=K^{\prime}$. Now, by Lemma 7.1,

$$
\begin{aligned}
n^{j} \mathbb{P}\left(A_{K, K^{\prime}}\right) & \leq C n^{j} \prod_{i=q}^{k+1} p_{i}^{\left(\begin{array}{c}
k+3 \\
i+1
\end{array}\right)}\left(\prod_{i=q}^{k+1} p_{i}^{\left(\begin{array}{c}
k+1 \\
i
\end{array}\right)}\right)^{j-k-3} \\
& =C n^{\tau_{k+2}(\boldsymbol{\alpha})+\alpha_{k+2}}\left(n^{1-\psi_{k+1}(\boldsymbol{\alpha})}\right)^{j-k-3} \leq n^{\tau_{k+2}(\boldsymbol{\alpha})+\alpha_{k+2}},
\end{aligned}
$$

and, by Proposition 3.1 and (2.12),

$$
\frac{n^{\tau_{k+2}(\boldsymbol{\alpha})+\alpha_{k+2}}}{\operatorname{Var}\left(f_{k}\right)} \leq C n^{\left(\tau_{k+1}(\boldsymbol{\alpha})-\tau_{k}(\boldsymbol{\alpha})\right)+\left(\tau_{q}(\boldsymbol{\alpha})-\tau_{k}(\boldsymbol{\alpha})\right)} \rightarrow 0, \quad n \rightarrow \infty .
$$

This completes the proof of (7.6) and, hence, of (4.4) in Theorem 4.2.

Finally, assuming (2.9) and (4.5), we establish tightness in the Skorohod $J_{1}$-topology. First of all, we already proved that under these assumptions, the convergence in (4.3) holds in the sense of weak convergence in the $J_{1}$-topology on $D[0, \infty)$. Fixing $T>0$ and choosing $m$ so large that $T / m \leq a / 4$ with $a$ defined in (2.3), we again consider a static multi-parameter simplicial complex $X\left([n], \mathbf{p}^{(1)}\right)$ and the corresponding $j$-face counts $f_{j}^{(1)}$, that were used for the proof of (6.7). By Proposition A.1 in the Appendix, all we have to do is to show that

$$
\left(\frac{\sum_{j=k+1}^{n-1}(-1)^{j} \beta_{j}(t)-\mathbb{E}\left(\sum_{j=k+1}^{n-1}(-1)^{j} \beta_{j}\right)}{\sqrt{\operatorname{Var}\left(f_{k}\right)}}, 0 \leq t \leq \frac{T}{m}\right) \rightarrow \mathbf{0}
$$

in probability in the $J_{1}$-topology. This will follow once we show that for every $\epsilon>0$,

$$
\mathbb{P}\left(\sup _{0 \leq t \leq T / m}\left|\sum_{j=k+1}^{n-1}(-1)^{j} \beta_{j}(t)-\mathbb{E}\left(\sum_{j=k+1}^{n-1}(-1)^{j} \beta_{j}\right)\right|>\epsilon \sqrt{\operatorname{Var}\left(f_{k}\right)}\right) \rightarrow 0, \quad n \rightarrow \infty .
$$

To this end, observe that by (4.5), for any $j \geq k+1$, we have

$$
E\left(f_{j}\right)=\mathcal{O}\left(n^{\tau_{k+1}(\boldsymbol{\alpha})}\right)=o\left(n^{\tau_{k}(\boldsymbol{\alpha})-\tau_{q}(\boldsymbol{\alpha}) / 2}\right)=o\left(\sqrt{\operatorname{Var}\left(f_{k}\right)}\right), \quad n \rightarrow \infty .
$$

Proceeding as in (6.11), while using $M(\tilde{\boldsymbol{\alpha}})$ defined in (3.7) and (6.8), we can bound the left hand side of (7.17) by

$$
\begin{aligned}
& \frac{2}{\epsilon \sqrt{\operatorname{Var}\left(f_{k}\right)}} \sum_{j=k+1}^{n-1} \mathbb{E}\left[\sup _{0 \leq t \leq T / m} f_{j}(t)\right] \leq \frac{2}{\epsilon \sqrt{\operatorname{Var}\left(f_{k}\right)}} \sum_{j=k+1}^{n-1} \mathbb{E}\left(f_{j}^{(1)}\right) \\
& \leq \frac{2}{\epsilon} \sum_{j=k+1}^{M(\tilde{\boldsymbol{\alpha}})-1} \frac{\prod_{i=q}^{j} 2^{\left({ }^{j+1}{ }_{i+1}\right)} \mathbb{E}\left(f_{j}\right)}{\sqrt{\operatorname{Var}\left(f_{k}\right)}}+\frac{2}{\epsilon} \sum_{j=M(\tilde{\boldsymbol{\alpha}})}^{\infty} \mathbb{E}\left(f_{j}^{(1)}\right) .
\end{aligned}
$$

The last term converges to 0 as $n \rightarrow \infty$ due to (7.18) and Corollary 3.4.

\section{Declaration of competing interest}

The authors declare that they have no known competing financial interests or personal relationships that could have appeared to influence the work reported in this paper.

\section{Acknowledgments}

The authors would like to thank the anonymous referee and the Associate Editor for their comments that lead to a substantial improvement of the paper. 


\section{Appendix}

\section{A.1. Analysis of the Betti numbers in lower dimensions}

We begin with introducing additional notions of connectivity. Given a simplicial complex $X$ and an $\ell$-dimensional simplex $\sigma$ in $X$, let the simplicial complex $\operatorname{lk}_{X}(\sigma):=\{\tau \in X: \sigma \cap \tau=$ $\emptyset, \sigma \cup \tau \in X$ \} denote the link of $\sigma$ in $X$. In other words, $\operatorname{lk}_{X}(\sigma)$ denotes the subcomplex of $X$ consisting of all simplices whose vertex support is disjoint from that of $\sigma$ but, together with $\sigma$, they form a simplex in $X$. If $X$ is pure $\ell$-dimensional and $\sigma$ is $(\ell-2)$-dimensional for some $\ell \geq 2$, then $\operatorname{lk}_{X}(\sigma)$ necessarily is a one-dimensional simplicial complex. We say that an $(\ell-1)$-face in $X$ is free if it is not contained in any of the $\ell$-faces in $X$. Given a graph $G$, we denote by $\lambda_{2}(G)$ the second smallest eigenvalue of the normalized graph Laplacian of $G$. We will use the cohomology vanishing theorem of Ballmann and Świątkowski [1]: if $X$ is a finite pure $\ell$-dimensional simplicial complex such that for every $(\ell-2)$-simplex $\sigma \in X$, the link $\mathrm{lk}_{X}(\sigma)$ is connected and has spectral gap $\lambda_{2}\left(\mathrm{k}_{X}(\sigma)\right)>1-1 / \ell$, then $H^{\ell-1}(X ; \mathbb{Q})=0$. In particular, $\beta_{\ell-1}(X)=0$.

Proposition A.1. Under the assumptions of Theorem 4.2,

$$
\left(\frac{\beta_{j}(t)-\mathbb{E}\left(\beta_{j}\right)}{\sqrt{\operatorname{Var}\left(f_{k}\right)}}, t \geq 0\right) \rightarrow \mathbf{0} \text { in } D[0, \infty)
$$

in probability as $n \rightarrow \infty$ for all $j=0,1, \ldots, k-1$, where $\mathbf{0}$ is the constant zero process.

Proof. If $k=1$ the claim is trivial, so assume that $k \geq 2$. We consider $j=k-1$ only; smaller dimensions can be treated in a similar way. Proposition A.1 will be established by combining a series of lemmas provided below. Let $F_{j}(t)$ be the number of free $j$-faces of $X([n], \mathbf{p} ; t)$, and $X_{k}(t)$ the $k$-skeleton of $X([n], \mathbf{p} ; t)$. For a $(k-2)$-face $\sigma$ in $X_{k}(t)$, write $L_{\sigma}(t):=\left|\mathrm{k}_{X_{k}(t)}(\sigma)\right|$, i.e., the number of vertices in the link of $\sigma$ in $X_{k}(t)$. We set $F_{j}:=F_{j}(0), X_{k}:=X_{k}(0)$, and $L_{\sigma}:=L_{\sigma}(0)$.

Consider the delayed renewal sequences defined in (2.5) corresponding to the stationary renewal processes $\left(\Delta_{i, A}, q \leq i \leq k, A \in \mathcal{W}_{i}\right)$. Enumerating the different arrival times, we denote the resulting sequence by $\eta_{1} \leq \eta_{2} \leq \cdots$, and set $\eta_{0}=0$. For $0<T<\infty$, we denote by $N(T)$ the number of these points in the interval $[0, T]$. Clearly, $\mathbb{E}(N(T))=\mathcal{O}\left(n^{k+1}\right)$ for every such $T$.

Lemma A.2. For each $0 \leq j \leq k-1$,

$$
\mathbb{E}\left(F_{j}\right)=o\left(e^{-n^{\epsilon}}\right), \quad n \rightarrow \infty,
$$

for some $\epsilon>0$.

Proof. A simple calculation shows that

$$
\mathbb{E}\left(F_{j}\right) \leq n^{\tau_{j}(\boldsymbol{\alpha})}\left(1-n^{-\psi_{j+1}(\boldsymbol{\alpha})}\right)^{n-j-1} .
$$

If $\psi_{j+1}(\boldsymbol{\alpha})=0$, the claim is trivial. Otherwise,

$$
\mathbb{E}\left(F_{j}\right) \leq C n^{\tau_{j}(\boldsymbol{\alpha})} e^{-n^{1-\psi_{j+1}(\boldsymbol{\alpha})}} .
$$

Since $\psi_{j+1}(\boldsymbol{\alpha}) \leq \psi_{k}(\boldsymbol{\alpha})<1$, the result follows. 


\section{Lemma A.3.}

$\mathbb{P}\left(X_{k}\right.$ is pure $)=1-o\left(e^{-n^{\epsilon}}\right), \quad n \rightarrow \infty$,

for some $\epsilon>0$.

Proof. By Lemma A.2,

$\mathbb{P}\left(X_{k}\right.$ is pure $)=\mathbb{P}\left(F_{j}=0, \quad j=0, \ldots, k-1\right)$

$$
\geq 1-\sum_{j=0}^{k-1} \mathbb{P}\left(F_{j} \geq 1\right) \geq 1-\sum_{j=0}^{k-1} \mathbb{E}\left(F_{j}\right)=1-o\left(e^{-n^{\epsilon}}\right) .
$$

Lemma A.4. Fix $\delta>0$. For $a(k-2)$-face $\sigma$ of $X_{k}$,

$$
\mathbb{P}\left(\frac{(1+\delta) \log L_{\sigma}}{L_{\sigma}}>p_{1}\right)=o\left(e^{-n^{\epsilon}}\right), \quad n \rightarrow \infty,
$$

for some $\epsilon>0$.

Proof. Note that

$$
1-\psi_{k-1}(\boldsymbol{\alpha})>\psi_{k}(\boldsymbol{\alpha})-\psi_{k-1}(\boldsymbol{\alpha}) \geq \alpha_{1},
$$

and $(1+\delta) x^{-1} \log x$ is decreasing for $x \geq e$. Therefore, if $L_{\sigma} \geq n^{1-\psi_{k-1}(\alpha)} / 2$, then

$$
\frac{(1+\delta) \log L_{\sigma}}{L_{\sigma}} \leq \frac{(1+\delta) \log \left(n^{1-\psi_{k-1}(\boldsymbol{\alpha})} / 2\right)}{n^{1-\psi_{k-1}(\boldsymbol{\alpha})} / 2}<n^{-\alpha_{1}}=p_{1}
$$

for large $n$. Hence, for large $n$,

$$
\mathbb{P}\left(\frac{(1+\delta) \log L_{\sigma}}{L_{\sigma}}>p_{1}\right) \leq \mathbb{P}\left(L_{\sigma}<\frac{n^{1-\psi_{k-1}(\boldsymbol{\alpha})}}{2}\right),
$$

and the claim follows from the basic properties of the binomial distribution because $L_{\sigma}$ has a binomial distribution with parameters $n-k+1$ and $n^{-\psi_{k-1}(\boldsymbol{\alpha})}$; see, e.g., Lemma 4.2 in [7].

Lemma A.5. For every $0<T<\infty$,

$$
\mathbb{P}\left(\sup _{0 \leq t \leq T} \beta_{k-1}(t) \neq 0\right)=\mathcal{O}\left(n^{-k-1}\right), \quad n \rightarrow \infty .
$$

Proof. By the cohomology vanishing theorem,

$$
\begin{aligned}
& \mathbb{P}\left(\sup _{0 \leq t \leq T} \beta_{k-1}(t)=0\right)=\mathbb{P}\left(\sup _{0 \leq t \leq T} \beta_{k-1}\left(X_{k}(t)\right)=0\right) \\
& =\mathbb{P}\left(\beta_{k-1}\left(X_{k}\left(\eta_{\ell}\right)\right)=0 \text { for } \ell=0,1, \ldots, N(T)\right) \\
& \geq \mathbb{P}\left(\beta_{k-1}\left(X_{k}\left(\eta_{\ell}\right)\right)=0 \text { for } \ell=0,1, \ldots, n^{2 k+2}, N(T) \leq n^{2 k+2}\right) \\
& \geq \mathbb{P}\left(\bigcap _ { \ell = 0 } ^ { n ^ { 2 k + 2 } } \left(\left\{\lambda_{2}\left(\operatorname{lk}_{X_{k}\left(\eta_{\ell}\right)}(\sigma)\right)>1-\frac{1}{k} \text { and } \operatorname{lk}_{X_{k}\left(\eta_{\ell}\right)}(\sigma)\right.\right.\right. \text { is connected } \\
& \left.\left.\left.\quad \text { for every }(k-2) \text {-face } \sigma \text { in } X_{k}\left(\eta_{\ell}\right)\right\} \cap\left\{X_{k}\left(\eta_{\ell}\right) \text { is pure }\right\}\right) \cap\left\{N(T) \leq n^{2 k+2}\right\}\right)
\end{aligned}
$$




$$
\begin{aligned}
\geq 1 & -\sum_{\ell=0}^{n^{2 k+2}}\left(\begin{array}{c}
n \\
k-1
\end{array}\right) \mathbb{P}\left(\lambda_{2}\left(\mathrm{lk}_{X_{k}\left(\eta_{\ell}\right)}\left(\sigma_{0}\right)\right) \leq 1-\frac{1}{k} \text { or } \operatorname{lk}_{X_{k}\left(\eta_{\ell}\right)}\left(\sigma_{0}\right) \text { is disconnected }\right) \\
& -\sum_{\ell=0}^{n^{2 k+2}} \mathbb{P}\left(X_{k}\left(\eta_{\ell}\right) \text { is not pure }\right)-\mathbb{P}\left(N(T)>n^{2 k+2}\right) .
\end{aligned}
$$

Here $\sigma_{0}$ is a fixed $(k-2)$-simplex. Clearly,

$$
\mathbb{P}\left(N(T)>n^{2 k+2}\right) \leq \frac{\mathbb{E}[N(T)]}{n^{2 k+2}}=\mathcal{O}\left(n^{-k-1}\right) ;
$$

so, by Lemma A.3 and the stationarity of $X_{k}$,

$$
\begin{aligned}
& \mathbb{P}\left(\sup _{0 \leq t \leq T} \beta_{k-1}(t)=0\right) \\
& \geq 1-n^{3 k+1} \mathbb{P}\left(\lambda_{2}\left(\operatorname{lk}_{X_{k}\left(\eta_{\ell}\right)}\left(\sigma_{0}\right)\right) \leq 1-\frac{1}{k} \text { or } \operatorname{lk}_{X_{k}\left(\eta_{\ell}\right)}\left(\sigma_{0}\right) \text { is disconnected }\right)-\mathcal{O}\left(n^{-k-1}\right) .
\end{aligned}
$$

Given $\sigma_{0} \in X_{k}$, we have by Lemma A.4 and its proof that, for some $\epsilon>0$,

$$
\begin{aligned}
& \mathbb{P}\left(\lambda_{2}\left(\mathrm{lk}_{X_{k}\left(\eta_{\ell}\right)}\left(\sigma_{0}\right)\right) \leq 1-\frac{1}{k} \text { or } \mathrm{lk}_{X_{k}\left(\eta_{\ell}\right)}\left(\sigma_{0}\right) \text { is disconnected }\right) \\
& =\mathbb{P}\left(\left\{\lambda_{2}\left(\mathrm{l}_{X_{k}\left(\eta_{\ell}\right)}\left(\sigma_{0}\right)\right) \leq 1-\frac{1}{k} \text { or } \mathrm{lk}_{X_{k}\left(\eta_{\ell}\right)}\left(\sigma_{0}\right) \text { is disconnected }\right\}\right. \\
& \left.\cap\left\{\frac{(1+\delta) \log L_{\sigma_{0}}}{L_{\sigma_{0}}} \leq p_{1}, L_{\sigma_{0}} \geq \frac{n^{1-\psi_{k-1}(\boldsymbol{\alpha})}}{2}\right\}\right)+o\left(e^{-n^{\epsilon}}\right) \\
& =\sum_{m=1}^{n-k+1} \mathbb{P}\left(\lambda_{2}\left(\mathrm{lk}_{X_{k}\left(\eta_{\ell}\right)}\left(\sigma_{0}\right)\right) \leq 1-\frac{1}{k} \text { or } \operatorname{lk}_{X_{k}\left(\eta_{\ell}\right)}\left(\sigma_{0}\right) \text { is disconnected } \mid L_{\sigma_{0}}=m\right) \\
& \quad \times \mathbb{1}\left\{\frac{(1+\delta) \log m}{m} \leq p_{1}, m \geq \frac{n^{1-\psi_{k-1}(\boldsymbol{\alpha})}}{2}\right\} \mathbb{P}\left(L_{\sigma}=m\right)+o\left(e^{-n^{\epsilon}}\right) .
\end{aligned}
$$

However, $\mathrm{lk}_{X_{k}}\left(\sigma_{0}\right) \mid L_{\sigma_{0}}=m$ has the law of the Erdös-Rényi graph with parameters $m$ and $p_{1}$; see Lemma 4.2 in [7]. Furthermore, in the range of $m$ we are considering, $p_{1} \geq \frac{(1+\delta) \log m}{m}$. It follows from the spectral gap theorem of Theorem 1.1 in [11] that for some $\delta$-dependent constant $C$,

$$
\mathbb{P}\left(\lambda_{2}\left(\mathrm{lk}_{X_{k}\left(\eta_{\ell}\right)}\left(\sigma_{0}\right)\right) \leq 1-\frac{1}{k} \text { or } \operatorname{lk}_{X_{k}\left(\eta_{\ell}\right)}\left(\sigma_{0}\right) \text { is disconnected } \mid L_{\sigma}=m\right) \leq C m^{-\delta} .
$$

We conclude that

$$
\begin{gathered}
\mathbb{P}\left(\lambda_{2}\left(\mathrm{lk}_{X_{k}\left(\eta_{\ell}\right)}\left(\sigma_{0}\right)\right) \leq 1-\frac{1}{k} \text { or } \mathrm{lk}_{X_{k}\left(\eta_{\ell}\right)}\left(\sigma_{0}\right) \text { is disconnected }\right) \\
\leq C\left(\frac{n^{1-\psi_{k-1}(\boldsymbol{\alpha})}}{2}\right)^{-\delta}+o\left(e^{-n^{\epsilon}}\right)=\mathcal{O}\left(n^{-\delta\left(1-\psi_{k-1}(\boldsymbol{\alpha})\right)}\right),
\end{gathered}
$$

and so

$$
\mathbb{P}\left(\sup _{0 \leq t \leq T} \beta_{k-1}(t)=0\right) \geq 1-\mathcal{O}\left(n^{3 k+1-\delta\left(1-\psi_{k-1}(\boldsymbol{\alpha})\right)}\right)-\mathcal{O}\left(n^{-k-1}\right) .
$$

As $1-\psi_{k-1}(\boldsymbol{\alpha})>0$, the claim follows by taking large enough $\delta>0$. 
We can now complete the proof of the proposition. Since $\operatorname{Var}\left(f_{k}\right) \rightarrow \infty$, we have for any $0<T<\infty$ and $\epsilon>0$, using Lemma A.5,

$$
\begin{aligned}
& \mathbb{P}\left(\sup _{0 \leq t \leq T}\left|\beta_{k-1}(t)-\mathbb{E}\left(\beta_{k-1}\right)\right|>\epsilon \sqrt{\operatorname{Var}\left(f_{k}\right)}\right) \leq \frac{2}{\epsilon} \mathbb{E}\left(\sup _{0 \leq t \leq T} \beta_{k-1}(t)\right) \\
& \leq \frac{2}{\epsilon} \mathbb{E}\left(\sup _{0 \leq t \leq T} f_{k-1}(t) \mathbb{1}\left\{\sup _{0 \leq t \leq T} \beta_{k-1}(t) \neq 0\right\}\right) \leq \frac{2}{\epsilon}\left(\begin{array}{l}
n \\
k
\end{array}\right) \mathbb{P}\left(\sup _{0 \leq t \leq T} \beta_{k-1}(t) \neq 0\right) \\
& =\frac{2}{\epsilon} \mathcal{O}\left(n^{-1}\right) \rightarrow 0, \quad n \rightarrow \infty,
\end{aligned}
$$

as required.

\section{A.2. Representation of the Betti number}

In this section we verify (7.3).

Proposition A.6. For $\ell \geq 1$,

$$
\beta_{\ell}(t)=\beta_{\ell}(X([n], \mathbf{p} ; t))=\sum_{j=\ell+2}^{n} \sum_{r \geq 1} \sum_{\sigma \subset[n],|\sigma|=j} r \eta_{\sigma}^{(j, r, \ell)}(t),
$$

where $\eta_{\sigma}^{(j, r, \ell)}(t)$ is the indicator function in (7.3).

Proof. For $\ell$-simplices $\sigma, \tau$ in $X([n], \mathbf{p} ; t)$, write $\sigma \sim \tau$ if they can be connected by a sequence of $\ell$-simplices $\sigma=\sigma_{0}, \sigma_{1}, \ldots, \sigma_{j-1}, \sigma_{j}=\tau$ such that $\operatorname{dim}\left(\sigma_{i} \cap \sigma_{i+1}\right)=\ell-1,0 \leq i \leq j-1$. Clearly $\sim$ is an equivalence relation. Consider the equivalence classes $\mathcal{G}_{1}, \ldots, \mathcal{G}_{N}$ associated with this relation. For each $i=1, \ldots, N$, let $X_{i}$ be the smallest subcomplex of $X([n], \mathbf{p} ; t)$ containing all the simplices for which some $\ell$-simplex in $\mathcal{G}_{i}$ is a face. Then $X_{i}$ is necessarily a maximal $\ell$-strongly connected subcomplex, such that $\operatorname{dim}\left(X_{i_{1}} \cap X_{i_{2}}\right) \leq \ell-2$ for any distinct $1 \leq i_{1} \neq i_{2} \leq N$. Let $X^{(N)}:=\bigcup_{i=1}^{N} X_{i}$ and let $X_{N+1}$ be a subcomplex of $X([n], \mathbf{p} ; t)$ containing all simplices in $X([n], \mathbf{p} ; t) \backslash X^{(N)}$. By construction $\operatorname{dim}\left(X_{N+1}\right) \leq \ell-1$ and $\operatorname{dim}\left(X_{N+1} \cap X^{(N)}\right) \leq \ell-2$. With this setup, establishing the claim of the proposition reduces to proving the following statements:

$$
\beta_{\ell}(X([n], \mathbf{p} ; t))=\beta_{\ell}\left(X^{(N)}\right),
$$

and

$$
\beta_{\ell}\left(X^{(N)}\right)=\sum_{i=1}^{N} \beta_{\ell}\left(X_{i}\right)
$$

Indeed, since $\sum_{i=1}^{N} \beta_{\ell}\left(X_{i}\right)$ in (A.3) is clearly equal to the right hand side of (A.1), our proof will be done once (A.2) and (A.3) are both established. For the proof of (A.2) we exploit the following Mayer-Vietoris exact sequence:

$$
\begin{aligned}
\cdots \rightarrow H_{\ell}\left(X^{(N)} \cap X_{N+1}\right) & \stackrel{\lambda_{\ell}}{\rightarrow} H_{\ell}\left(X^{(N)}\right) \oplus H_{\ell}\left(X_{N+1}\right) \rightarrow H_{\ell}(X([n], \mathbf{p} ; t)) \\
& \rightarrow H_{\ell-1}\left(X^{(N)} \cap X_{N+1}\right) \stackrel{\lambda_{\ell-1}}{\rightarrow} H_{\ell-1}\left(X^{(N)}\right) \oplus H_{\ell-1}\left(X_{N+1}\right) \rightarrow \ldots,
\end{aligned}
$$

where $H_{\ell}$ represents the homology group of order $\ell$, and $\lambda_{\ell}=\left(\lambda_{\ell}^{(1)}, \lambda_{\ell}^{(2)}\right)$ denotes the homomorphism induced by the inclusions $X^{(N)} \cap X_{N+1} \hookrightarrow X^{(N)}$ and $X^{(N)} \cap X_{N+1} \hookrightarrow X_{N+1}$. 
An elementary rank calculation (see e.g., Lemma 2.3 in [22]) yields

$$
\beta_{\ell}(X([n], \mathbf{p} ; t))=\beta_{\ell}\left(X^{(N)}\right)+\beta_{\ell}\left(X_{N+1}\right)+\operatorname{rank}\left(\operatorname{ker} \lambda_{\ell}\right)+\operatorname{rank}\left(\operatorname{ker} \lambda_{\ell-1}\right)-\beta_{\ell}\left(X^{(N)} \cap X_{N+1}\right) .
$$

Since $\operatorname{dim}\left(X_{N+1}\right) \leq \ell-1$ and $\operatorname{dim}\left(X^{(N)} \cap X_{N+1}\right) \leq \ell-2$, we have that

$$
H_{\ell}\left(X_{N+1}\right) \cong 0, \quad H_{\ell}\left(X^{(N)} \cap X_{N+1}\right) \cong 0, \quad H_{\ell-1}\left(X^{(N)} \cap X_{N+1}\right) \cong 0 .
$$

In particular, ker $\lambda_{\ell}$ and $\operatorname{ker} \lambda_{\ell-1}$ are both trivial. Combining all these observations we obtain (A.2).

We now turn to deriving (A.3). The statement is trivial for $N=1$. If $N>1$, we denote $X^{(j)}:=\bigcup_{i=1}^{j} X_{i}$ and prove that $\beta_{\ell}\left(X^{(j)}\right)=\sum_{i=1}^{j} \beta_{\ell}\left(X_{i}\right)$ for $j=1, \ldots, N$ inductively. Once again, the case $j=1$ is trivial, so suppose for induction that $\beta_{\ell}\left(X^{(j-1)}\right)=\sum_{i=1}^{j-1} \beta_{\ell}\left(X_{i}\right)$ for some $1 \leq j<N$. We consider another Mayer-Vietoris exact sequence, given by

$$
\begin{aligned}
\cdots \rightarrow H_{\ell}\left(X^{(j-1)} \cap X_{j}\right) & \stackrel{\nu_{\ell}}{\rightarrow} H_{\ell}\left(X^{(j-1)}\right) \oplus H_{\ell}\left(X_{j}\right) \rightarrow H_{\ell}\left(X^{(j)}\right) \\
& \rightarrow H_{\ell-1}\left(X^{(j-1)} \cap X_{j}\right) \stackrel{\nu_{\ell-1}}{\rightarrow} H_{\ell-1}\left(X^{(j-1)}\right) \oplus H_{\ell-1}\left(X_{j}\right) \rightarrow \ldots,
\end{aligned}
$$

where $v_{\ell}, v_{\ell-1}$ are group homomorphisms analogous to the earlier situation. Since $\operatorname{dim}\left(X^{(j-1)} \cap\right.$ $\left.X_{j}\right) \leq \ell-2$, the same rank computation as above gives us

$$
\begin{aligned}
& \beta_{\ell}\left(X^{(j)}\right)=\beta_{\ell}\left(X^{(j-1)}\right)+\beta_{\ell}\left(X_{j}\right)+\operatorname{rank}\left(\operatorname{ker} v_{\ell}\right)+\operatorname{rank}\left(\operatorname{ker} v_{\ell-1}\right)-\beta_{\ell}\left(X^{(j-1)} \cap X_{j}\right) \\
& =\sum_{i=1}^{j} \beta_{\ell}\left(X_{i}\right)
\end{aligned}
$$

completing the induction step.

\section{References}

[1] W. Ballmann, J. Świątkowski, On $L^{2}$-cohomology and property (T) for automorphism groups of polyhedral cell complexes, Geom. Funct. Anal. 7 (1997) 615-645.

[2] A. Barbour, M. Karoński, A. Ruciński, A central limit theorem for decomposable random variables with applications to random graphs, J. Combin. Theory Ser. B 47 (1989) 125-145.

[3] P. Billingsley, Convergence of Probability Measures, second ed., Wiley, New York, 1999.

[4] A. Costa, M. Farber, Random Simplicial Complexes. Configuration Spaces, in: Springer INdAM Series, vol. 14, Springer, Cham, 2016.

[5] A. Costa, M. Farber, Large random simplicial complexes, III: the critical dimension, J. Knot Theory Ramif. 26 (2017) 1740010, 1-26.

[6] P. Erdös, A. Rényi, On random graphs. I, Publ. Math. Debrecen 6 (1959) 290-297.

[7] C. Fowler, Homology of multi-parameter random simplicial complexes, Discrete Comput. Geom. 62 (1) (2019) $87-127$.

[8] N. Fraiman, S. Mukherjee, G. Thoppe, The Bulk and the Extremes of Minimal Spanning Acycles and Persistence Diagrams of Random Complexes. arXiv:2012.14122.

[9] M. Hino, S. Kanazawa, Asymptotic behavior of lifetime sums for random simplicial complex processes, J. Math. Soc. Japan, 71 (3) 765-804.

[10] Y. Hiraoka, T. Shirai, Minimum spanning acycle and lifetime of persistent homology in the linial-meshulam process, Random Struct. Algorithms 51 (2) (2017) 315-340.

[11] C. Hoffman, M. Kahle, E. Paquette, Spectral gaps of random graphs and applications, International Mathematics Research Notices (2019).

[12] M. Kahle, Topology of random clique complexes, Discrete Math. 309 (2009) 1658-1671.

[13] M. Kahle, Sharp vanishing thresholds for cohomology of random flag complexes, Ann. of Math. 179 (2014) 1085-1107. 
[14] M. Kahle, E. Meckes, Limit theorems for betti numbers of random simplicial complexes, Homology, Homotopy Appl. 15 (2013) 343-374, Erratum: 18 (2016), 129-142.

[15] N. Linial, R. Meshulam, Homological connectivity of random 2-complexes, Combinatorica 26 (2006) $475-487$.

[16] R. Meshulam, N. Wallach, Homological connectivity of random k-complexes, Random Struct. Algorithms 34 (2009) 408-417.

[17] B. Pittel, A random graph with a subcritical number of edges, Trans. Amer. Math. Soc. 309 (1988) 51-75.

[18] S. Resnick, Adventures in Stochastic Processes, Birkhäuser, Boston, 1992.

[19] S. Ross, Stochastic Processes, second ed., Wiley, New York, 1996.

[20] P. Skraba, G. Thoppe, D. Yogeshwaran, Randomly weighted $d$-complexes: Minimal spanning acycles and persistence diagrams, Electron. J. Combin. 27 (2) (2020).

[21] G. Thoppe, D. Yogeshwaran, R. Adler, On the evolution of topology in dynamic clique complexes, Adv. Appl. Probab. 48 (2016) 989-1014.

[22] D. Yogeshwaran, E. Subag, R. Adler, Random geometric complexes in thermodynamic regime, Probab. Theory Related Fields 167 (2017) 107-142. 